\title{
Constructing Solutions to the Björling Problem for Isothermic Surfaces by Structure Preserving Discretization
}

\author{
Ulrike Bücking and Daniel Matthes
}

\begin{abstract}
In this article, we study an analog of the Björling problem for isothermic surfaces (that are a generalization of minimal surfaces): given a regular curve $\gamma$ in $\mathbb{R}^{3}$ and a unit normal vector field $n$ along $\gamma$, find an isothermic surface that contains $\gamma$, is normal to $n$ there, and is such that the tangent vector $\gamma^{\prime}$ bisects the principal directions of curvature. First, we prove that this problem is uniquely solvable locally around each point of $\gamma$, provided that $\gamma$ and $n$ are real analytic. The main result is that the solution can be obtained by constructing a family of discrete isothermic surfaces (in the sense of Bobenko and Pinkall) from data that is read off from $\gamma$, and then passing to the limit of vanishing mesh size. The proof relies on a rephrasing of the Gauss-Codazzi-system as analytic Cauchy problem and an in-depth-analysis of its discretization which is induced from the geometry of discrete isothermic surfaces. The discrete-to-continuous limit is carried out for the Christoffel and the Darboux transformations as well.
\end{abstract}

\section{Introduction}

Isothermic surfaces are among the most classical objects in differential geometry: these are surfaces that admit a conformal parametrization along curvature lines, see Definition 1. Like various particular geometries-special coordinate systems, minimal surfaces, surfaces of constant curvature-they have been introduced and intensively studied in the second half of the 19th century [9, 24]. Also, like the many of these classical objects, they have been "rediscovered" in the 1990s, both in connection with integrable systems and in the context of discrete differential

\footnotetext{
U. Bücking

Inst. für Mathematik, Technische Universität Berlin,

Straße des 17. Juni 136, 10623 Berlin, Germany

e-mail: buecking@math.tu-berlin.de

D. Matthes ( $\square)$

Zentrum Mathematik - M8, Technische Universität München,

Boltzmannstr. 3, D-85747 Garching bei München, Germany

e-mail: matthes@ma.tum.de 

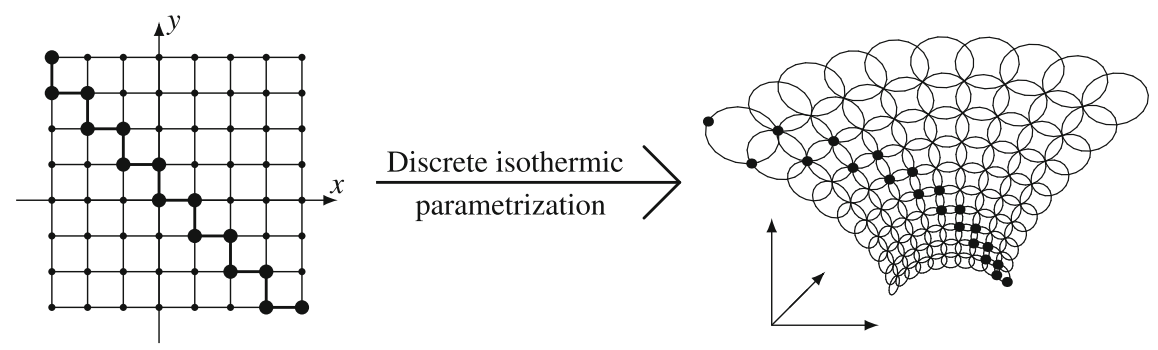

Fig. 1 Initial zig-zag on a discrete isothermic surface

geometry. The first description of isothermic surfaces as soliton surfaces is found in [11]. The first definition for discrete isothermic surfaces was made shortly after in [3]. In the most simple case, these are immersions of $\mathbb{Z}^{2}$ into $\mathbb{R}^{3}$ such that the vertices of each elementary quadrilateral are conformally equivalent to the corners of a planar square, see Definition 3.

This discrete surface class, its transformations and invariances has been studied e.g. in $[7,8,20]$. A systematic presentation of the theory of isothermic surfaces in the context of Möbius geometry can be found in [15]. Finally, we refer to [4] for a detailed overview on discrete isothermic surfaces as part of discrete differential geometry, including historical remarks.

Despite the manifold results on (classical) isothermic surfaces and the related equations, the fundamental question about their construction from suitably chosen data has apparently been left open. On the one hand, the machinery of integrable systems enables one to construct a rich variety of "solitonic" isothermic surfaces [11]. But on the other hand, nothing seems to be known about the well-posedness of an initial or boundary value problem for the Gauss-Codazzi-equations in general. The latter form a PDE system (cf. Eq. (5)) which contains both elliptic and hyperbolic equations. ${ }^{1}$ The appearance of an elliptic equation suggests that data for the surface boundary should be prescribed, as it is done for minimal surfaces for example. The hyperbolic equations, on the other hand, suggest to provide data for two curvature lines instead, like in the case of level surfaces in triply orthogonal systems [1]. Neither of the two approaches seems promising for the coupled system.

In contrast, there is a canonical way to pose an initial value problem for a discrete isothermic surface. One prescribes the vertices in $\mathbb{R}^{3}$ for a "zig-zag"-curve in parameter space as indicated in Fig. 1. For vertices in general position, these data can be extended to a discrete isothermic surface in a unique way. In fact, all vertices on the discrete surface are easily obtained inductively from the prescribed data.

In this paper, we formulate and prove solvability of a Björling problem for real analytic isothermic surfaces. And we prove that the solution can be obtained as the continuous limit of discrete isothermic surfaces.

\footnotetext{
${ }^{1}$ The system of Gauss-Codazzi-equations can be simplified to Calapso's equation [8], which is a single scalar fourth order PDE, but unfortunately of indefinite type.
} 
The classical Björling problem is to find a minimal surface that touches a given curve in $\mathbb{R}^{3}$ along prescribed tangent planes. This problem has been solved in general, see [13]. An extension of the Björling problem to surfaces of constant mean curvature has been posed (and solved) in [5]. A natural formulation of the Björling problem in the yet more general class of isothermic surfaces reads as follows.

Problem 1 Given a regular curve $\gamma$ in $\mathbb{R}^{3}$, and two mutually orthogonal unit vector fields $v, w$ along $\gamma$, neither of which is tangent to $\gamma$ at any point. Find an isothermic surface $S$ containing $\gamma$ such that $v$ and $w$ are the principal directions of curvature at each point of $\gamma$.

We believe that this problem is solvable locally provided that $\gamma, v$ and $w$ are all real analytic. Indeed, the non-tangency of the vector fields allows to give a reformulation in terms of a non-characteristic Cauchy problem. However, we do not address the Björling problem in this full generality here, but stick to the following restricted setting, where we do not prescribe two tangent vector fields $v$ and $w$ individually, but only a two-dimensional tangent plane:

Problem 2 Given a regular curve $\gamma$ in $\mathbb{R}^{3}$, and a unit vector field $n$ that is orthogonal to the tangent vectors $\gamma^{\prime}$ at each point. Find an isothermic surface $S$ containing $\gamma$ such that at each point of $\gamma$, the vector $n$ is normal to $S$, and each of the two directions of principal curvature encloses an angle $\pi / 4$ with $\gamma^{\prime}$.

As a corollary of the results presented here, it follows that this problem is uniquely solvable for real analytic $\gamma$ and $n$, at least locally around each point of $\gamma$. Existence and uniqueness of a real analytic isothermic surface $S$ for given data is the minor result of this paper, see Theorem 1 . The main result is that the real analytic data can be "sampled" with a mesh width $\epsilon>0$ in a suitable way such that the discrete isothermic surfaces $S^{\epsilon}$ constructed from the discrete data converge in $C^{1}$ to $S$. The precise formulation is given in Theorem 2.

It is remarkable that naive numerical experiments suggest that such an approximation result might not be true. It was already noted in [3] that discrete isothermic surfaces depend very sensitively on their initial data. The limit $\epsilon \rightarrow 0$ is delicate, and inappropriate choices of the initial zig-zag cause the sequence $S^{\epsilon}$ to diverge rapidly. In fact, even the possibility to construct any sequence of discrete isothermic surfaces that approximates a given smooth one is not obvious. Discrete isothermic surfaces are one of many examples of a discretized geometric structure for which the passage back to the original continuous structure needs a highly non-trivial approximation result, the proof of which is analysis-based and goes far beyond elementary geometric considerations. Further such non-trivial convergence results are available, for instance, for discrete surfaces of constant negative Gaussian curvature [2], for discrete triply orthogonal systems [1], and, most importantly, for circle patterns $[6,21,22]$ as approximations to conformal maps.

The core of our convergence proof is a stability analysis of the discrete GaussCodazzi system that we derive for discrete isothermic surfaces. We show that the solution to the discrete Gauss-Codazzi equations with sampled data as initial condition remains close to the solution of the classical Gauss-Codazzi system for the same 
(continuous) initial data. In a second step, this implies proximity of the respective discrete and continuous surfaces. We are able to quantify the approximation error in terms of the supremum-distance between analytic functions on complex domains: it is linear in the mesh size. In fact, we conjecture that this result is sub-optimal, and second-order approximation should be provable, using a more refined analysis and a more careful approximation of the data.

The techniques used in the proof are similar to those employed by one of the authors [17] to prove convergence of circle patterns to conformal maps. The geometric situation for isothermic surfaces, however, is much more complicated, and the structure of the Gauss-Codazzi system is much more complex than the CauchyRiemann equations. The proof of stability relies on estimates for the solution of analytic Cauchy problems in scales of Banach spaces. These estimates have been developed - in the classical, non-discretized setting - in Nagumo's famous article [18] as part of the existence proof for analytic Cauchy problems. Here, we shall rather use Nirenberg's [19] version of these estimates. For an overview over the history of analytic Cauchy problems and the related estimates, see the beautiful article of Walter [23].

Note that the convergence proof here is more direct than the one in [17]. While the latter was based on purely discrete considerations, the current proof uses semidiscrete techniques: a-somewhat artificial-extension of the discrete functions to continuous domains allows to formulate estimates more easily. The main simplification, however, is that we separate the proofs for existence of a classical solution and its approximation by discrete solutions.

The paper is organized as follows. In Sect. 2 we formulate the Gauss-Codazzisystem for smooth isothermic surfaces in the framework of analytic Cauchy problems and prove unique local solvability of the Björling problem by the CauchyKowalevskaya theorem. In Sect. 3 we derive an analogous system of difference equations for discrete isothermic surfaces. For appropriate initial conditions, the convergence of the discrete solutions to the corresponding smooth ones is proven in Sect. 4. Then, in Sect. 5 we explain how to discretize the Björling initial data appropriately, and prove convergence of the discrete surfaces to the respective smooth one. Finally, in Sect. 6, the convergence result is extended to Christoffel and Darboux transformations.

\section{Smooth Isothermic Surfaces}

We start by summarizing basic properties of smooth isothermic surfaces and proving our first result on the local solvability of the Björling problem. 
Fig. 2 Relation between the coordinates $(x, y)$ and $(\xi, \eta)$

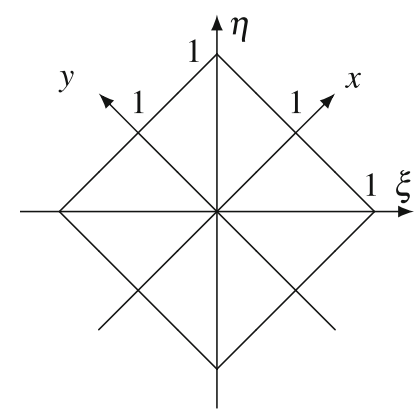

\subsection{Coordinates and Domains}

For concise statements and proofs, we need to work with two different coordinate systems $(\xi, \eta)$ and $(x, y)$ on $\mathbb{R}^{2}$ simultaneously. These coordinates are related to each other by

$$
\xi=\frac{x-y}{2}, \quad \eta=\frac{x+y}{2} \Leftrightarrow x=\eta+\xi, \quad y=\eta-\xi,
$$

see Fig. 2. Accordingly, the partial derivatives transform as follows:

$$
\partial_{\xi}=\partial_{x}-\partial_{y}, \quad \partial_{\eta}=\partial_{x}+\partial_{y}
$$

Observe in particular that

$$
\partial_{\xi}^{2}+\partial_{\eta}^{2}=2\left(\partial_{x}^{2}+\partial_{y}^{2}\right)
$$

It will be convenient to consider $(\xi, \eta)$ as the "basic" coordinates and $(x, y)$ as the auxiliary ones. More precisely: in the rare cases that we need to specify explicitly the arguments of a function $g: \Omega \rightarrow \mathbb{R}$ defined on a domain $\Omega \subset \mathbb{R}^{2}$, then we shall write $g(\xi, \eta)$ for the value of $g$ at the point with coordinates $x=\eta+\xi$ and $y=\eta-\xi$.

For further reference, define for $r \geq h>0$ the domains

$$
\Omega(r \mid h)=\left\{(\xi, \eta) \in \mathbb{R}^{2} ;|\xi|+|\eta| \leq r,-h<\eta \leq h\right\} .
$$

In the $(x, y)$-coordinates, $\Omega(r \mid h)$ is a axes-parallel square of side length $2 r$, centered at the origin, that is cut off at the top-right and bottom-left corners.

\subsection{Definition and Equations}

By abuse of notation, we use the term "(parametrized) surface" for a smooth and non-degenerate map $F: \Omega(r \mid h) \rightarrow \mathbb{R}^{3}$. Here non-degeneracy means that the vector 
fields $F_{x}$ and $F_{y}$ are linearly independent. Every such surface comes with a smooth normal map $N: \Omega(r \mid h) \rightarrow \mathbb{S}^{2}$, given by

$$
N=\frac{F_{x} \times F_{y}}{\left\|F_{x} \times F_{y}\right\|} .
$$

Definition $1 F: \Omega(r \mid h) \rightarrow \mathbb{R}^{3}$ is a (parametrized) isothermic surface, if

(1) $F$ is conformal, i.e., there exists a conformal factor $u: \Omega(r \mid h) \rightarrow \mathbb{R}$ such that

$$
\left\|F_{x}\right\|^{2}=\left\|F_{y}\right\|^{2}=e^{2 u}, \quad\left\langle F_{x}, F_{y}\right\rangle=0,
$$

(2) $F$ parametrizes along curvature lines, i.e., the normal map $N: \Omega(r \mid h) \rightarrow \mathbb{S}^{2}$ satisfies

$$
\left\langle F_{x y}, N\right\rangle=0 .
$$

The quantities $\mathfrak{k}, \mathfrak{l}: \Omega(r \mid h) \rightarrow \mathbb{R}$ in

$$
-\left\langle N_{x}, F_{x}\right\rangle=e^{u} \mathfrak{k}, \quad-\left\langle N_{y}, F_{y}\right\rangle=e^{u} \mathfrak{l},
$$

are the (scaled) principal curvatures.

Remark 1 The genuine principal curvature functions are given by $e^{-u \mathfrak{k}}$ and $e^{-u} \mathfrak{l}$. The quantities $\mathfrak{k}$ and $\mathfrak{l}$ are better suited for the calculations below.

The next result is classical.

Lemma 1 Assume that an isothermic surface $F: \Omega(r \mid h) \rightarrow \mathbb{R}^{3}$ is given. Then the conformal factor $u$ and the scaled curvatures $\mathfrak{k}, \mathfrak{l}$ satisfy the Gauss-Codazzi equations

$$
-\left(u_{x x}+u_{y y}\right)=\mathfrak{k l}, \quad \mathfrak{l}_{x}=\mathfrak{k} u_{x}, \quad \mathfrak{k}_{y}=\mathfrak{l} u_{y} .
$$

Conversely, if functions $u, \mathfrak{k}, \mathfrak{l}: \Omega(r \mid h) \rightarrow \mathbb{R}$ satisfy the system (5), then there exists an isothermic surface $F: \Omega(r \mid h) \rightarrow \mathbb{R}$ that has $u$ as its conformal factor and has scaled curvatures $\mathfrak{k}, \mathfrak{l}$. Moreover, $F$ is uniquely determined up to Euclidean motions.

We briefly recall the proof, since we shall need some of the calculations later.

Proof (Sketch) For a given isothermic surface $F: \Omega(r \mid h) \rightarrow \mathbb{R}^{3}$, introduce the adapted frame

$$
\Psi:=\left(e^{-u} F_{x}, e^{-u} F_{y}, N\right): \Omega(r \mid h) \rightarrow \operatorname{SO}(3)
$$

and define the transition matrices $U, V: \Omega(r \mid h) \rightarrow$ so(3) implicitly by

$$
\Psi_{x}=\Psi U, \quad \Psi_{y}=\Psi V
$$


Using the defining properties of the isothermic parametrization, one easily obtains the following explicit expressions for $U$ and $V$ :

$$
U=\left(\begin{array}{ccc}
0 & u_{y} & -\mathfrak{k} \\
-u_{y} & 0 & 0 \\
\mathfrak{k} & 0 & 0
\end{array}\right), \quad V=\left(\begin{array}{ccc}
0 & -u_{x} & 0 \\
u_{x} & 0 & -\mathfrak{l} \\
0 & \mathfrak{l} & 0
\end{array}\right)
$$

The compatibility condition $U_{y}-V_{x}=U V-V U$ implies the equations in (5).

Conversely, if $u, \mathfrak{k}, \mathfrak{l}$ satisfy (5), then the matrix functions $U, V: \Omega(r \mid h) \rightarrow \operatorname{so}(3)$ defined by (7) satisfy compatibility condition $U_{y}-V_{x}=U V-V U$. Consequently, one can define a further matrix function $\Psi=\left(\Psi_{1}, \Psi_{2}, \Psi_{3}\right): \Omega(r \mid h) \rightarrow \mathrm{SO}(3)$ as solution to the system (6). Clearly, the solution $\Psi$ is uniquely determined by its value $\Psi(0) \in \mathrm{SO}(3)$ at $(x, y)=0$. The particular form of $U$ and $V$ imply that

$$
\partial_{x}\left(e^{u} \Psi_{2}\right)=\partial_{y}\left(e^{u} \Psi_{1}\right)
$$

which further implies the existence of a map $F: \Omega(r) \rightarrow \mathbb{R}^{3}$ such that

$$
\partial_{x} F=e^{u} \Psi_{1} \quad \text { and } \quad \partial_{y} F=e^{u} \Psi_{2}
$$

The map $F$ is non-degenerate, and it is uniquely determined by its value $F(0) \in \mathbb{R}^{3}$ at $(x, y)=0$. Clearly $\Psi$ is an adapted frame for the surface defined by $F$, whose normal vector field is given by $\Psi_{3}$. It follows directly from (8) that $F$ is conformal (3). The property (4) is a further direct consequence of (6) and the special form of $U$ and $V$ from (7).

A different form of the Gauss-Codazzi equations (5) is needed in the following. Remind the relation between the coordinates $(x, y)$ and $(\xi, \eta)$ by $x=\eta+\xi$ and $y=\eta-\xi$ in Sect.2.1. Introduce auxiliary functions $v, w: \Omega(r \mid h) \rightarrow \mathbb{R}$ by

$$
v=\frac{1}{2} u_{\xi}, \quad w=\frac{1}{2} u_{\eta}
$$

Further recall (2). Then the Gauss-Codazzi system (5) attains the form

$$
\begin{aligned}
v_{\eta} & =w_{\xi}, \\
w_{\eta} & =-v_{\xi}-\mathfrak{k l}, \\
\mathfrak{k}_{y} & =\mathfrak{l}(w-v), \\
\mathfrak{l}_{x} & =\mathfrak{k}(w+v) .
\end{aligned}
$$




\subsection{Local Solution of the Björling Problem}

The following result implies local solvability of (the restricted version of) the Björling problem for isothermic surfaces, with real analytic data. To see the equivalence to Problem 2 stated in the introduction, observe that the conformal parametrization $F$ of an isothermic surface and our coordinates in (1) are such that the images of $\{x=$ const $\}$ and of $\{y=$ const $\}$ are mapped to curvature lines under $F$, whereas the tangent to each curve $\xi \mapsto F(\xi, \eta)$ is always at an angle of $\pi / 4$ to both curvature directions.

Theorem 1 Let an analytic and regular curve $f:(-r, r) \rightarrow \mathbb{R}^{3}$ and an analytic normal unit vector field $n:(-r, r) \rightarrow \mathbb{S}^{2}$ be given, that is $\left\langle f^{\prime}, n\right\rangle \equiv 0$. Then, for some $h>0$ with $h \leq r$, there exists a unique analytic isothermic surface $F: \Omega(r \mid h) \rightarrow \mathbb{R}^{3}$ such that $F$ and its normal vector field $N$ satisfy

$$
F(\xi, 0)=f(\xi), \quad N(\xi, 0)=n(\xi) \text { for all } \xi \in(-r, r)
$$

Remark 2 The original Björling problem consists in finding a minimal surface in $\mathbb{R}^{3}$ that touches a given curve along prescribed tangent planes. See [5] for an extension to constant mean curvature surfaces. Our problem is a bit different since (13) implies in addition that the tangential vector to the data curve is everywhere at angle $\pi / 4$ with the directions of principal curvature, see (14). Such additional restrictions are expected to guarantee unique solvability of the Björling problem in the much larger class of isothermic surfaces.

Proof (of Theorem 1) If there exists an isothermic surface $F: \Omega(r \mid h) \rightarrow \mathbb{R}^{3}$ with the properties (13), then

$$
f^{\prime}(\xi)=F_{\xi}(\xi, 0)=F_{x}(\xi, 0)-F_{y}(\xi, 0)
$$

at every $\xi \in(-r, r)$, and in particular

$$
\left\|f^{\prime}(\xi)\right\|^{2}=\left\|F_{x}(\xi, 0)\right\|^{2}+\left\|F_{y}(\xi, 0)\right\|^{2}-\left\langle F_{x}(\xi, 0), F_{y}(\xi, 0)\right\rangle=2 e^{2 u(\xi, 0)} .
$$

It follows that $f$ and $n$ determine both the conformal factor $u$ and the adapted frame $\Psi=\left(e^{-u} F_{x}, e^{-u} F_{y}, N\right): \Omega(r \mid h) \rightarrow \mathrm{SO}(3)$ uniquely on $\eta=0$; denote the corresponding functions by $u^{0}:(-r, r) \rightarrow \mathbb{R}$ and $\Psi^{0}:(-r, r) \rightarrow \mathrm{SO}(3)$, respectively.

Next, introduce functions $v^{0}, w^{0}, \mathfrak{k}^{0}, \mathfrak{l}^{0}:(-r, r) \rightarrow \mathbb{R}$ by

$$
\left(u^{0}\right)^{\prime}=2 v^{0} u^{0}, \quad\left(\Psi^{0}\right)^{\prime}=\Psi^{0}\left(\begin{array}{ccc}
0 & 2 w^{0} & -\mathfrak{k}^{0} \\
-2 w^{0} & 0 & \mathfrak{l}^{0} \\
\mathfrak{k}^{0} & -\mathfrak{l}^{0} & 0
\end{array}\right) .
$$

The line $\{\eta=0\}=\{x+y=0\}$ is obviously non-characteristic for the system of equations (9)-(12). Hence, the Cauchy-Kowalevskaya theorem applies in this situation. For some sufficiently small $h>0$, there exists a unique analytic solution 
$v, w, \mathfrak{k}, \mathfrak{l}: \Omega(r \mid h) \rightarrow \mathbb{R}$ to (9)-(12) with the initial conditions $v^{0}, w^{0}, \mathfrak{k}^{0}, \mathfrak{l}^{0}$ at $\eta=0$. Since (9) is a compatibility condition for the linear system

$$
u_{\xi}=2 v, \quad u_{\eta}=2 w
$$

there exists a unique analytic solution $u: \Omega(r \mid h) \rightarrow \mathbb{R}$ with $u=u^{0}$ for $\eta=0$. The triple $(u, \mathfrak{k}, \mathfrak{l})$ satisfies $(5)$. Lemma 1 guarantees the existence of a unique isothermic surface $F: \Omega(r \mid h) \rightarrow \mathbb{R}^{3}$ with $u$ as conformal factor, with scaled principle curvatures $\mathfrak{k}$ and $\mathfrak{l}$, and with the normalizations

$$
F(0)=f(0), N(0)=n(0), F_{x}(0)-F_{y}(0)=f^{\prime}(0)
$$

Analyticity of $F$ is clear from its construction in the proof. To see that $F$ attains the initial data (13), first observe that an adapted frame $\Psi$ necessarily satisfies $\Psi_{\xi}=$ $\Psi_{x}-\Psi_{y}=\Psi(U-V)$, and so $\Psi=\Psi^{0}$ on $\eta=0$, thanks to (7) and (15), (16). In particular, we have that $\Psi_{3}(\xi, 0)=N(\xi)$. And further, $F_{\xi}=\Psi_{1}-\Psi_{2}=\Psi_{1}^{0}-\Psi_{2}^{0}$ implies $F=f$ on $\eta=0$.

Concerning uniqueness: $f$ and $\Psi^{0}$ determine the initial data $\left(v^{0}, w^{0}, \mathfrak{k}^{0}, \mathfrak{l}^{0}\right)$ for (9)(12)_-and hence also its solution $(v, w, \mathfrak{k}, \mathfrak{l})$ - uniquely. Invoking again Lemma 1 , it follows that $F$ with the normalization (16) is unique as well.

\section{Discrete Isothermic Surfaces}

Throughout this section, we assume that some (small) parameter $\epsilon>0$ is given, which quantifies the average mesh width of the considered discrete isothermic surfaces. We introduce the abbreviation

$$
z^{*}=\sqrt{1-\epsilon^{2} z^{2}}
$$

for arbitrary quantities $z$, assuming that $|\epsilon z|<1$.

\subsection{Coordinates and Domains}

Recall that we are working with the two coordinate systems from (1) simultaneously, $(\xi, \eta)$ being the "basic" coordinates and $(x, y)$ being the "auxiliary" ones. Introduce the associated shift-operators $T_{x}, T_{y}, T_{\xi}, T_{\eta}$ by

$$
\begin{array}{ll}
T_{x}(\xi, \eta)=\left(\xi+\frac{\epsilon}{4}, \eta+\frac{\epsilon}{4}\right), & T_{\xi}(\xi, \eta)=\left(\xi+\frac{\epsilon}{2}, \eta\right) \\
T_{y}(\xi, \eta)=\left(\xi-\frac{\epsilon}{4}, \eta+\frac{\epsilon}{4}\right), & T_{\eta}(\xi, \eta)=\left(\xi, \eta+\frac{\epsilon}{2}\right) .
\end{array}
$$


By slight abuse of notation, we shall use the same symbols for the associated contravariant shifts of functions $f: \Omega(r \mid h) \rightarrow \mathbb{R}$, i.e., $T_{x} f:=f \circ T_{x}$ etc. The associated central difference quotient operators are defined by

$$
\begin{aligned}
\delta_{x} f & =\frac{1}{\epsilon}\left(T_{x} f-T_{x}^{-1} f\right), & \delta_{\xi} f & =\frac{1}{\epsilon}\left(T_{\xi} f-T_{\xi}^{-1} f\right) \\
\delta_{y} f & =\frac{1}{\epsilon}\left(T_{y} f-T_{y}^{-1} f\right), & \delta_{\eta} f & =\frac{1}{\epsilon}\left(T_{\eta} f-T_{\eta}^{-1} f\right) .
\end{aligned}
$$

It is a notorious inconvenience in discrete differential geometry that the various quantities which are derived from discrete geometric objects are associated to different natural domains of definition. To account for that, we need to single out specific subdomains inside our basic domain $\Omega(r \mid h)$ : let

$$
\Omega^{[x] \epsilon}(r \mid h)=\Omega(r \mid h) \cap T_{x} \Omega(r \mid h) \cap T_{x}^{-1} \Omega(r \mid h),
$$

be the natural domain of definition for $\delta_{x} f$, when $f$ is defined on $\Omega(r \mid h)$. Likewise, we define $\Omega^{[y] \epsilon}(r \mid h)$. The domain

$$
\Omega^{[x y] \epsilon}(r \mid h)=\Omega\left(r-\frac{\epsilon}{2} \mid h-\frac{\epsilon}{2}\right)
$$

is such that the mixed difference quotient $\delta_{x} \delta_{y} f$ is well-defined there; notice that $\delta_{\xi} f$ and $\delta_{\eta} f$ are well-defined on $\Omega^{[x y] \epsilon}(r \mid h)$. In the same spirit, we introduce $\Omega^{[x x y] \epsilon}(r \mid h)$ as domain for $\delta_{x}^{2} \delta_{y} f$ etc. For each point $\zeta \in \Omega^{[x y] \epsilon}(r \mid h)$, we say that the four points $T_{\xi} \zeta, T_{\eta} \zeta, T_{\xi}^{-1} \zeta$ and $T_{\eta}^{-1} \zeta$ form an elementary $\epsilon$-square.

\subsection{Definition of Discrete Isothermic Surfaces}

In this section, we give a variant of the definition for discrete isothermic surfaces from [3], which is well-suited for the passage to the continuum limit. First, we need auxiliary notation.

Definition 2 Four points $p_{1}, \ldots, p_{4} \in \mathbb{R}^{3}$ form a (non-degenerate) conformal square iff they lie on a circle, but no three of them are on a line, they are cyclically ordered, ${ }^{2}$ and their mutual distances are related by

$$
\left\|p_{1}-p_{2}\right\| \cdot\left\|p_{3}-p_{4}\right\|=\left\|p_{1}-p_{4}\right\| \cdot\left\|p_{2}-p_{3}\right\| \text {. }
$$

Remark 3 The name refers to the fact that $p_{1}, \ldots, p_{4}$ form a (non-degenerate) conformal square if and only if there is a Möbius transformation of $\mathbb{R}^{3}$ which takes these points to the corners of the unit square, $(0,0,0),(1,0,0),(1,1,0)$ and $(0,1,0)$,

\footnotetext{
${ }^{2}$ Cyclic ordering means that walking around the circle either clockwise or anti-clockwise, one passes $p_{1}, p_{2}, p_{3}$ and $p_{4}$ in that order, see Fig. 3 (left).
} 
Fig. 3 Conformal squares and the association of quantities to lattice points
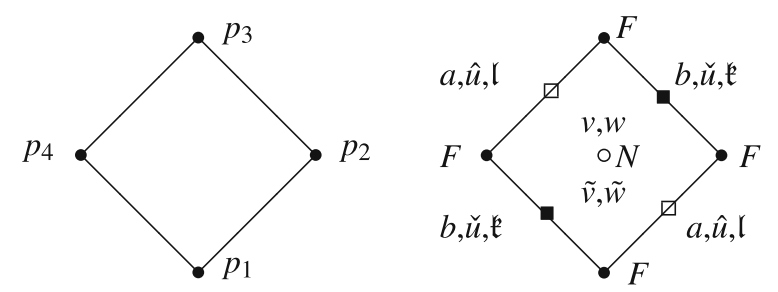

respectively. Notice that the non-degeneracy condition is important for the equivalence, since certain point configurations on a straight line can be Möbius transformed into the unit square as well.

Alternatively, one could define conformal squares by saying that $p_{1}$ to $p_{4}$ have cross-ratio equal to minus one, either in the sense of quaternions, see for example [15], or after identification of these points with complex numbers in their common plane. Again, non-degeneracy is important for equivalence of the definitions.

The following is an easy exercise in elementary geometry.

Lemma 2 For any given three points $p_{1}, p_{2}, p_{3} \in \mathbb{R}^{3}$ (with ordering) that are not collinear (and in particular pairwise distinct), there exists precisely one fourth point $p_{4} \in \mathbb{R}^{3}$ that completes the conformal square. Moreover, the coordinates of $p_{4}$ depend analytically on those of $p_{1}, p_{2}$ and $p_{3}$.

We are now going to state the main definition, namely the one for discrete isothermic surfaces. Originally [3], discrete surfaces have been introduced as particular immersed lattices in $\mathbb{R}^{3}$. Having the continuous limit in mind, we give a slightly different definition, which describes a continuous immersion in $\mathbb{R}^{3}$, corresponding to a two-parameter family of lattices.

Definition 3 A map $F^{\epsilon}: \Omega(r \mid h) \rightarrow \mathbb{R}^{3}$ is called (the parametrization of) a $\epsilon$ discrete isothermic surface, if elementary $\epsilon$-squares are mapped to conformal squares in $\mathbb{R}^{3}$.

Remark 4 Since no continuity is required for $F^{\epsilon}: \Omega(r \mid h) \rightarrow \mathbb{R}^{3}$, one can think of it - at this point-for instance as the piecewise constant extension of a map $\tilde{F}^{\epsilon}$ : $\Lambda^{\epsilon}(r \mid h) \rightarrow \mathbb{R}^{3}$ that is only defined on a suitable lattice $\Lambda^{\epsilon}(r \mid h) \subset \Omega(r \mid h)$, e.g. on

$$
\Lambda^{\epsilon}(r \mid h)=\left\{(\xi, \eta) \in \Omega(r \mid h) ; \frac{\xi}{\epsilon}+\frac{\eta}{\epsilon} \in \mathbb{Z}\right\} .
$$

Alternatively, one can say that $F^{\epsilon}: \Omega(r \mid h) \rightarrow \mathbb{R}^{3}$ is a discrete isothermic surface, if and only if the four vectors

$$
T_{y} \delta_{x} F^{\epsilon}, T_{x} \delta_{y} F^{\epsilon}, T_{y}^{-1} \delta_{x} F^{\epsilon}, T_{x}^{-1} \delta_{y} F^{\epsilon}
$$


always lie in one common plane and satisfy

$$
\left\|T_{y} \delta_{x} F^{\epsilon}\right\|\left\|_{T_{y}^{-1}} \delta_{x} F^{\epsilon}\right\|=\left\|T_{x} \delta_{y} F^{\epsilon}\right\|\left\|T_{x}^{-1} \delta_{y} F^{\epsilon}\right\| .
$$

Note that this identity is a discrete replacement for the relation $\left\|F_{x}\right\|^{2}=\left\|F_{y}\right\|^{2}$ on smooth conformally parametrized surfaces.

\subsection{The Discrete Björling Problem}

We introduce the analog of the Björling problem for $\epsilon$-discrete isothermic surfaces. In contrast to its continuous counterpart, its solution is immediate. First, we need some more notation to formulate conditions on the data.

Definition 4 A function $f^{\epsilon}: \Omega(r \mid h) \rightarrow \mathbb{R}^{3}$ is said to be non-degenerate if neither any of the point triples

$$
\left(T_{\xi}^{-1} f^{\epsilon}, T_{\eta}^{-1} f^{\epsilon}, T_{\xi} f^{\epsilon}\right)(\xi, \eta)
$$

nor any of the point triples

$$
\left(T_{\xi}^{-1} f^{\epsilon}, T_{\eta} f^{\epsilon}, T_{\xi} f^{\epsilon}\right)\left(\xi^{\prime}, \eta^{\prime}\right)
$$

are collinear, where $(\xi, \eta),\left(\xi^{\prime}, \eta^{\prime}\right) \in \Omega(r \mid h)$ are arbitrary points such that these respective values of $f^{\epsilon}$ are defined. If collinearities occur, then $f^{\epsilon}$ is called degenerate.

Definition 5 We call a function $f^{\epsilon}: \Omega\left(r \mid \frac{\epsilon}{2}\right) \rightarrow \mathbb{R}^{3}$ Björling data for the construction of an $\epsilon$-discrete isothermic surface if it is non-degenerate.

Proposition 1 Let $\bar{h}$ and $\epsilon>0$ with $r>\bar{h}>\frac{\epsilon}{2}$ and Björling data $f^{\epsilon}$ be given. Then, there exists some maximal $h \in\left(\frac{\epsilon}{2}, \bar{h}\right]$ and a unique $\epsilon$-discrete isothermic surface $F^{\epsilon}$ : $\Omega(r \mid h) \rightarrow \mathbb{R}^{3}$ such that $F^{\epsilon}=f^{\epsilon}$ on $\Omega\left(r \mid \frac{\epsilon}{2}\right)$. Here maximal has to be understood as follows: either $h=\bar{h}$, or the restriction of $F^{\epsilon}$ to $\Omega\left(r \mid h-\frac{\epsilon}{2}\right)$ is degenerate.

Proof The proof is a direct application of Lemma 2: from the data $f^{\epsilon}$ given on $\Omega\left(r \mid \frac{\epsilon}{2}\right)$, one directly calculates the values of $F^{\epsilon}$ on $\Omega(r \mid \epsilon)$. These are then extended to $\Omega\left(r \mid 3 \frac{\epsilon}{2}\right)$ in the next step, and so on. The procedure works as long as no degeneracies occur. 


\subsection{Discrete Quantities and Basic Relations}

Let some discrete isothermic surface $F^{\epsilon}: \Omega(r \mid h) \rightarrow \mathbb{R}^{3}$ be given. Below, we introduce quantities that play an analogous role for $F^{\epsilon}$ as $u, \mathfrak{k}, \mathfrak{l}$ etc. do for $F$. Figure 3 (right) indicates, on which lattices these respective quantities live.

Define the discrete conformal factors $\hat{u}: \Omega^{[x] \epsilon}(r \mid h) \rightarrow \mathbb{R}$ and $\check{u}: \Omega^{[y] \epsilon}(r \mid h) \rightarrow$ $\mathbb{R}$, respectively, by

$$
e^{\hat{u}}=\left\|\delta_{x} F^{\epsilon}\right\|, \quad e^{\check{u}}=\left\|\delta_{y} F^{\epsilon}\right\| .
$$

Thanks to the property (19) of discrete isothermic surfaces, these seemingly different quantities are related to each other by the identity

$$
T_{x} \check{u}+T_{x}^{-1} \check{u}=T_{y} \hat{u}+T_{y}^{-1} \hat{u}
$$

that holds on $\Omega^{[x y] \epsilon}(r \mid h)$. We may thus unambiguously define the discrete derivatives $v, w: \Omega^{[x y] \epsilon}(r \mid h) \rightarrow \mathbb{R}$ of the conformal factor by

$$
v=\frac{T_{x} \check{u}-T_{y} \hat{u}}{\epsilon}=\frac{T_{y}^{-1} \hat{u}-T_{x}^{-1} \check{u}}{\epsilon}, \quad w=\frac{T_{x} \check{u}-T_{y}^{-1} \hat{u}}{\epsilon}=\frac{T_{y} \hat{u}-T_{x}^{-1} \check{u}}{\epsilon} .
$$

Next, define the discrete unit tangent vectors $a: \Omega^{[x] \epsilon}(r \mid h) \rightarrow \mathbb{S}^{2}$ and $b: \Omega^{[y] \epsilon}(r \mid h)$ $\rightarrow \mathbb{S}^{2}$, respectively, by

$$
a=e^{-\hat{u}} \delta_{x} F^{\epsilon}, \quad b=e^{-\check{u}} \delta_{y} F^{\epsilon} .
$$

Since conformal squares are planar, there is a natural notion of normal field $N$ : $\Omega^{[x y] \epsilon}(r \mid h) \rightarrow \mathbb{S}^{2}$, namely

$$
N=\frac{T_{y} \delta_{x} F^{\epsilon} \times T_{x} \delta_{y} F^{\epsilon}}{\left\|T_{y} \delta_{x} F^{\epsilon} \times T_{x} \delta_{y} F^{\epsilon}\right\|}
$$

With the help of the discrete orthonormal frame $(a, b, N)$, we introduce the discrete scaled principal curvatures $\mathfrak{k}: \Omega^{[x x y] \epsilon}(r \mid h) \rightarrow \mathbb{R}$ and $\mathfrak{l}: \Omega^{[x y y] \epsilon}(r \mid h) \rightarrow \mathbb{R}$, respectively, by

$$
\epsilon \mathfrak{k}=-\left\langle T_{x}^{-1} N \times T_{x} N, b\right\rangle, \quad \epsilon \mathfrak{l}=\left\langle T_{y}^{-1} N \times T_{y} N, a\right\rangle .
$$

Note that $\epsilon \mathfrak{k}$ and $\epsilon \mathfrak{l}$ are equal to $\sin \angle\left(T_{x}^{-1} N, T_{x} N\right)$ and to $\sin \angle\left(T_{y}^{-1} N, T_{y} N\right)$, respectively, with the signs chosen to maintain consistency with the continuous quantities.

Finally, to facilitate the calculations below, we need two more discrete functions $\tilde{v}, \tilde{w}: \Omega^{[x y] \epsilon}(r \mid h) \rightarrow \mathbb{R}$, given by 


$$
\begin{gathered}
\epsilon \tilde{v}=\frac{\left\langle T_{y} \delta_{x} F^{\epsilon}, T_{x}^{-1} \delta_{y} F^{\epsilon}\right\rangle}{\left\|T_{y} \delta_{x} F^{\epsilon}\right\| T_{x}^{-1} \delta_{y} F^{\epsilon} \|}=-\frac{\left\langle T_{y}^{-1} \delta_{x} F^{\epsilon}, T_{x} \delta_{y} F^{\epsilon}\right\rangle}{\left\|T_{y}^{-1} \delta_{x} F^{\epsilon}\right\|\left\|T_{x} \delta_{y} F^{\epsilon}\right\|}, \\
\epsilon \tilde{w}=\frac{\left\langle T_{y} \delta_{x} F^{\epsilon}, T_{x} \delta_{y} F^{\epsilon}\right\rangle}{\left\|T_{y} \delta_{x} F^{\epsilon}\right\|\left\|T_{x} \delta_{y} F^{\epsilon}\right\|}=-\frac{\left\langle T_{y}^{-1} \delta_{x} F^{\epsilon}, T_{x}^{-1} \delta_{y} F^{\epsilon}\right\rangle}{\left\|T_{y}^{-1} \delta_{x} F^{\epsilon}\right\|\left\|T_{x}^{-1} \delta_{y} F^{\epsilon}\right\|} .
\end{gathered}
$$

The equalities follow since opposite angles in a conformal square sum up to $\pi$. The two pairs $(v, w)$ and $(\tilde{v}, \tilde{w})$ are just different representations of the same geometric information.

Lemma 3 There is a one-to-one correspondence between the pairs $(v, w)$ and $(\tilde{v}, \tilde{w})$ of functions. Specifically, recalling the *-notation introduced in (17),

$$
\sinh (\epsilon v)=\epsilon \frac{\tilde{v} \tilde{w}^{*}}{\tilde{v}^{*}} \text { and } \sinh (\epsilon w)=\epsilon \frac{\tilde{w} \tilde{v}^{*}}{\tilde{w}^{*}} .
$$

Moreover, the pair $(v, \tilde{w})$ uniquely determines the pair $(\tilde{v}, w)$, and vice versa.

Proof This is a general statement about four geometric quantities defined for conformal squares. It thus suffices to consider a single conformal square with vertices

$$
p_{1}=T_{\eta}^{-1} F^{\epsilon}, p_{2}=T_{\xi} F^{\epsilon}, p_{3}=T_{\eta} F^{\epsilon}, p_{4}=T_{\xi}^{-1} F^{\epsilon} \text {. }
$$

The respective four real numbers $v, w, \tilde{v}, \tilde{w}$ are given by

$$
\begin{gathered}
e^{\epsilon v}=\frac{\left\|p_{2}-p_{1}\right\|}{\left\|p_{1}-p_{4}\right\|}=\frac{\left\|p_{3}-p_{2}\right\|}{\left\|p_{4}-p_{3}\right\|}, \quad e^{\epsilon w}=\frac{\left\|p_{3}-p_{2}\right\|}{\left\|p_{2}-p_{1}\right\|}=\frac{\left\|p_{3}-p_{4}\right\|}{\left\|p_{4}-p_{1}\right\|}, \\
\epsilon \tilde{v}=\cos \left(\angle p_{1} p_{2} p_{3}\right)=-\cos \left(\angle p_{3} p_{4} p_{1}\right), \quad \epsilon \tilde{w}=\cos \left(\angle p_{2} p_{3} p_{4}\right)=-\cos \left(\angle p_{4} p_{1} p_{2}\right) .
\end{gathered}
$$

Observe that

$$
\begin{aligned}
\left\|p_{3}-p_{2}\right\|^{2}+\left\|p_{1}-p_{2}\right\|^{2}-2\left\langle p_{3}-p_{2}, p_{1}-p_{2}\right\rangle & =\left\|p_{3}-p_{4}\right\|^{2} \\
+ & \left\|p_{1}-p_{4}\right\|^{2}-2\left\langle p_{3}-p_{4}, p_{1}-p_{4}\right\rangle
\end{aligned}
$$

since both expressions are equal to $\left\|p_{3}-p_{1}\right\|^{2}$. Divide by $\left\|p_{3}-p_{2}\right\|^{2}$ and use the definitions of $v, w, \tilde{v}, \tilde{w}$ to obtain, after simplification, that

$$
1+e^{-2 \epsilon w}-2 \epsilon \tilde{v} e^{-\epsilon w}=e^{-2 \epsilon v}\left(1+e^{-2 \epsilon w}+2 \epsilon \tilde{v} e^{-\epsilon w}\right) .
$$

The analogous considerations with $\left\|p_{4}-p_{2}\right\|^{2}$ in place of $\left\|p_{3}-p_{1}\right\|^{2}$ give (24) with $\tilde{w}$ in place of $\tilde{v}$, and with the roles of $w$ and $v$ exchanged. Clearly, these equations are uniquely solvable for $(\tilde{v}, \tilde{w})$ in terms of $(v, w)$ :

$$
\epsilon \tilde{v}=\tanh (\epsilon v) \cosh (\epsilon w), \quad \epsilon \tilde{w}=\tanh (\epsilon w) \cosh (\epsilon v)
$$


Note that in particular

$$
\epsilon^{2} \tilde{v} \tilde{w}=\sinh (\epsilon v) \sinh (\epsilon w)
$$

To derive (23) from here, take the square of the equations in (25), and express $\cosh ^{2}$ and $\tanh ^{2}$ in terms of $\sinh ^{2}$ only. Then use (26) to eliminate $\sinh ^{2}(\epsilon w)$ from the first equation and $\sinh ^{2}(\epsilon v)$ from the second one. This yields

$$
\sinh ^{2}(\epsilon v)=\left(\epsilon \frac{\tilde{v} \tilde{w}^{*}}{\tilde{v}^{*}}\right)^{2}, \quad \sinh ^{2}(\epsilon w)=\left(\epsilon \frac{\tilde{w} \tilde{v}^{*}}{\tilde{w}^{*}}\right)^{2} .
$$

Now take the square root, bearing in mind that $v, \tilde{v}$ have the same sign, and $w, \tilde{w}$ have the same sign by (25).

Finally, to calculate $\tilde{v}$ from a given $(v, \tilde{w})$ using the first relation in (23), it suffices to invert the (strictly increasing) function $\tilde{v} \mapsto \tilde{v} / \tilde{v}^{*}$. Then, knowing $\tilde{v}$ and $\tilde{w}$, the value of $w$ can be obtained from the second relation in (23).

Recall that all discrete quantities defined above depend on the parameter $\epsilon$. To stress this fact, we will in the following use the superscript $\epsilon$.

For later reference, we draw some first consequences of the definitions above. Specifically, we summarize the relations between the geometric quantities $\left(a^{\epsilon}, b^{\epsilon}\right.$, $\left.\hat{u}^{\epsilon}, \check{u}^{\epsilon}\right)$, and, of course, to $F^{\epsilon}$ itself, to the more abstract quantities $\left(v^{\epsilon}, w^{\epsilon}, \mathfrak{k}^{\epsilon}, \mathfrak{l}^{\epsilon}\right)$ that satisfy the Gauss-Codazzi system (31)-(34). These relations can be seen as a discrete analog of the frame equations (6) and (7).

Lemma 4 On $\Omega^{[x x y y] \epsilon}(r \mid h)$, one has

$$
\begin{aligned}
& \delta_{y} F^{\epsilon}=\exp \left(\hat{u}^{\epsilon}\right) a^{\epsilon}, \quad \delta_{x} F^{\epsilon}=\exp \left(\check{u}^{\epsilon}\right) b^{\epsilon}, \\
& \delta_{y} \hat{u}^{\epsilon}=w^{\epsilon}-v^{\epsilon}, \quad \delta_{x} \check{u}^{\epsilon}=w^{\epsilon}+v^{\epsilon}, \\
& \delta_{y} a^{\epsilon}=\left[\frac{\left(\tilde{v}^{\epsilon}\right)^{*}}{\left(\tilde{w}^{\epsilon}\right)^{*}} \tilde{w}^{\epsilon}-\tilde{v}^{\epsilon}\right] T_{x}^{-1} b^{\epsilon}+\frac{1}{\epsilon}\left[\frac{\left(\tilde{v}^{\epsilon}\right)^{*}}{\left(\tilde{w}^{\epsilon}\right)^{*}}-1\right] T_{y}^{-1} a^{\epsilon}, \\
& \delta_{x} b^{\epsilon}=\left[\frac{\left(\tilde{v}^{\epsilon}\right)^{*}}{\left(\tilde{w}^{\epsilon}\right)^{*}} \tilde{w}^{\epsilon}-\tilde{v}^{\epsilon}\right] T_{y}^{-1} a^{\epsilon}+\frac{1}{\epsilon}\left[\frac{\left(\tilde{v}^{\epsilon}\right)^{*}}{\left(\tilde{w}^{\epsilon}\right)^{*}}-1\right] T_{x}^{-1} b^{\epsilon} .
\end{aligned}
$$

Proof The two equations in (28) are obtained by rearranging the identities in (20). For the derivation of (29), one makes the ansatz

$$
T_{y} a^{\epsilon}=\mu_{a} T_{y}^{-1} a^{\epsilon}+\mu_{b} T_{x}^{-1} b^{\epsilon} .
$$

Such a representation of $T_{y} a^{\epsilon}$ must exist since elementary squares are mapped to (flat) quadrilaterals by $F^{\epsilon}$. The coefficients $\mu_{a}$ and $\mu_{b}$ can be determined by solving the system of equations 
$1=\left\|T_{y} a^{\epsilon}\right\|^{2}=\mu_{a}^{2}+\mu_{b}^{2}-2 \epsilon \mu_{a} \mu_{b} \tilde{w}^{\epsilon}, \quad \epsilon \tilde{v}^{\epsilon}=\left\langle T_{y} a^{\epsilon}, T_{x}^{-1} b^{\epsilon}\right\rangle=-\epsilon \mu_{a} \tilde{w}^{\epsilon}+\mu_{b}$.

The analogous ansatz—with the roles of $a^{\epsilon}$ and $b^{\epsilon}$ interchanged—leads to (30).

\subsection{Discrete Gauss-Codazzi System}

This section is devoted to derive a discrete version of the Gauss-Codazzi equations (9)-(12). The following definition is needed to classify the difference between the continuous and the discrete system.

Definition 6 A family $\left(h_{\epsilon}\right)_{\epsilon>0}$ of real functions on respective domains $D_{\epsilon} \subset \mathbb{R}^{n}$ is called asymptotically analytic on $\mathbb{C}^{n}$ if the following is true. For every $M>0$, there is an $\epsilon(M)>0$ such that each $h_{\epsilon}$ with $0<\epsilon<\epsilon(M)$ extends from $D_{\epsilon}$ to a complex-analytic function $\tilde{h}_{\epsilon}: \mathbb{D}_{M}^{n} \rightarrow \mathbb{C}$ on the $n$-dimensional complex multi-disc

$$
\mathbb{D}_{M}^{n}=\left\{z=\left(z_{1}, \ldots, z_{n}\right) \in \mathbb{C}^{n}|| z_{j} \mid<M \text { for each } j=1, \ldots, n\right\} .
$$

And the extensions $\tilde{h}_{\epsilon}$ are bounded on $\mathbb{D}_{M}^{n}$, uniformly in $0<\epsilon<\epsilon(M)$.

The prototypical example for a family $\left(h_{\epsilon}\right)_{\epsilon>0}$ that is asymptotically analytic on $\mathbb{C}$ is given by $h_{\epsilon}(z)=1 / z^{*}=\left(1-\epsilon^{2} z^{2}\right)^{-1 / 2}$. It is further easily seen that also the functions $g_{\epsilon}=\epsilon^{-2}\left(h_{\epsilon}-1\right)$ form such a family; this is a very strong way of saying that $h_{\epsilon}=1+\mathscr{O}\left(\epsilon^{2}\right)$.

Proposition 2 There are four families $\left(h_{1, \epsilon}\right)_{\epsilon>0}, \ldots,\left(h_{4, \epsilon}\right)_{\epsilon>0}$ of asymptotically analytic functions on $\mathbb{C}^{8}$ for which the following is true: let any $\epsilon$-discrete isothermic surface $F^{\epsilon}: \Omega(r \mid h) \rightarrow \mathbb{R}^{3}$ be given, and define the functions $v^{\epsilon}, w^{\epsilon}, \mathfrak{k}^{\epsilon}, \mathfrak{l}^{\epsilon}$ accordingly. Then the following system of discrete equations is satisfied on $\Omega^{[x y] \epsilon}(r \mid h)$ :

$$
\begin{aligned}
\delta_{\eta} v^{\epsilon} & =\delta_{\xi} w^{\epsilon}, \\
\delta_{\eta} w^{\epsilon} & =\delta_{\xi} v^{\epsilon}-\left(T_{y}^{-1} \mathfrak{k}^{\epsilon}\right)\left(T_{x}^{-1} \mathfrak{l}^{\epsilon}\right)+\epsilon h_{2}^{\epsilon}\left(T \theta^{\epsilon}\right), \\
\delta_{y} \mathfrak{k}^{\epsilon} & =\left(T_{x}^{-1} \mathfrak{l}^{\epsilon}\right)\left(T_{\eta}^{-1} w^{\epsilon}-T_{\xi} v^{\epsilon}\right)+\epsilon^{2} h_{3}^{\epsilon}\left(T \theta^{\epsilon}\right) \\
\delta_{x} \mathfrak{l}^{\epsilon} & =\left(T_{y}^{-1} \mathfrak{k}^{\epsilon}\right)\left(T_{\eta}^{-1} w^{\epsilon}+T_{\xi}^{-1} v^{\epsilon}\right)+\epsilon h_{4}^{\epsilon}\left(T \theta^{\epsilon}\right),
\end{aligned}
$$

where the $h_{j}^{\epsilon}$ are evaluated on

$$
T \theta^{\epsilon}=\left(T_{\xi} v^{\epsilon}, T_{\xi} w^{\epsilon}, T_{\xi}^{-1} v^{\epsilon}, T_{\xi}^{-1} w^{\epsilon}, T_{\eta}^{-1} v^{\epsilon}, T_{\eta}^{-1} w^{\epsilon}, T_{y}^{-1} \mathfrak{k}^{\epsilon}, T_{x}^{-1} \mathfrak{l}^{\epsilon}\right) .
$$

Remark 5 Equations (31)-(34) are explicit in $\eta$-direction in the sense that they express the "unknown" quantities $T_{\eta} v^{\epsilon}, T_{\eta} w^{\epsilon}, T_{y} \mathfrak{k}^{\epsilon}$ and $T_{x}{ }^{\epsilon}$ in terms of the "given" eight quantities summarized in $T \theta^{\epsilon}$. 
Fig. 4 Four elementary squares with discrete quantities for the Cauchy problem
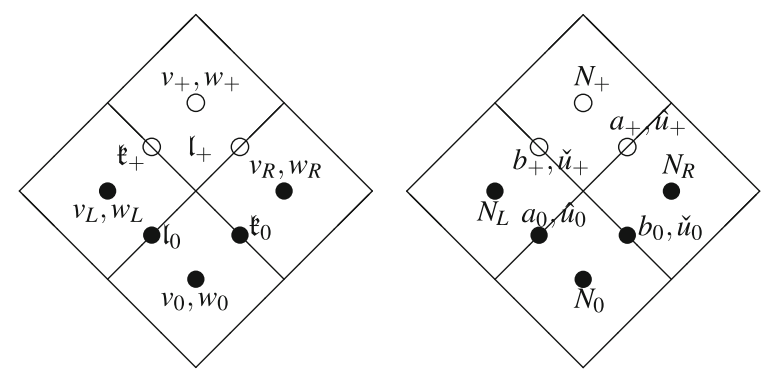

The rest of this section is devoted to the proof of Proposition 2. Since $\epsilon>0$ is fixed in the derivation of (31)-(34), we shall omit the superscript $\epsilon$ on the occurring quantities.

For the derivation of (31)-(34), one can obviously work locally: it suffices to fix some point in $\Omega^{[x x y y] \epsilon}(r \mid h)$ and to consider the eight values of $v, w$ on the midpoints of the four elementary squares incident to that vertex, and the four values of $\mathfrak{k}, \mathfrak{l}$ on the respective connecting edges.

The setup is visualized in Fig. 4. The "unknown" quantities $v_{+}, w_{+}$and $\mathfrak{k}_{+}, \mathfrak{l}_{+}$are marked by o, the "given" quantities $v_{0}, w_{0}, v_{L}, w_{L}, v_{R}, w_{R}$ and $\mathfrak{k}_{0}, \mathfrak{l}_{0}$ are marked by $\bullet$. To facilitate the calculations, we also assume that values for $a_{0}, b_{0}, \hat{u}_{0}, \check{u}_{0}, N_{0}, N_{L}$, $N_{R}$ are given; and then obtain the values of $a_{+}, b_{+}, \hat{u}_{+}, \check{u}_{+}, N_{+}$, see Fig. 4 right. Naturally, the final formulas for $v_{+}, w_{+}$and $\mathfrak{k}_{+}, \mathfrak{l}_{+}$will be independent of these quantities.

\subsubsection{Derivation of Equation (31)}

Compare the following two alternative ways to calculate $\hat{u}_{+}$, the logarithmic length of the edge separating the right and the top plaquettes, from $\hat{u}_{0}$, the logarithmic length of the edge between the plaquettes at bottom and left:

$$
e^{\epsilon \hat{u}_{0}} e^{\epsilon v_{0}} e^{\epsilon w_{R}}=e^{\epsilon \hat{u}+}=e^{\epsilon \hat{u}_{0}} e^{\epsilon W_{L}} e^{\epsilon v_{+}}
$$

holds by property (20) of the functions $v$ and $w$. Take the logarithm to obtain (31).

\subsubsection{Derivation of Equation (32)}

First recall that by Lemma 3, there is a one-to-one correspondence between $(v, w)$ and $(\tilde{v}, \tilde{w})$, so we can assume that values for $\left(\tilde{v}_{0}, \tilde{w}_{0}\right),\left(\tilde{v}_{L}, \tilde{w}_{L}\right),\left(\tilde{v}_{R}, \tilde{w}_{R}\right)$ are given as well. Using that $N_{R}$ is the normalized cross product $a_{+} \times b_{0}$, it is elementary to derive the following representation of $a_{+}$: 


$$
a_{+}=\epsilon \tilde{v}_{R} b_{0}+\tilde{v}_{R}^{*}\left(b_{0} \times N_{R}\right)
$$

Taking the scalar product with $N_{0}$, one obtains

$$
\left\langle a_{+}, N_{0}\right\rangle=\tilde{v}_{R}^{*}\left\langle b_{0}, N_{R} \times N_{0}\right\rangle=\epsilon \tilde{v}_{R}^{*} \mathfrak{k}_{0} .
$$

Hence $a_{+}$can be expanded in the basis $a_{0}, b_{0}$ and $N_{0}$ as follows:

$$
a_{+}=\mu_{a} a_{0}+\mu_{b} b_{0}+\epsilon \tilde{v}_{R}^{*} \mathfrak{k}_{0} N_{0}
$$

with some real coefficients $\mu_{a}$ and $\mu_{b}$ to be determined. Calculating the square norm on both sides gives

$$
1=\mu_{a}^{2}+\mu_{b}^{2}+2 \epsilon \tilde{w}_{0} \mu_{a} \mu_{b}+\epsilon^{2}\left(\tilde{v}_{R}^{*}\right)^{2} \mathfrak{k}_{0}^{2},
$$

and the scalar product with $b_{0}$ yields

$$
\epsilon \tilde{v}_{R}=\epsilon \tilde{w}_{0} \mu_{a}+\mu_{b}
$$

Use (38) to eliminate $\mu_{b}$ from (37), then solve for $\mu_{a}$. This gives

$$
\mu_{a}=\frac{\tilde{v}_{R}^{*} \mathfrak{k}_{0}^{*}}{\tilde{w}_{0}^{*}}, \quad \mu_{b}=\epsilon \tilde{v}_{R}-\epsilon \frac{\tilde{v}_{R}^{*} \mathfrak{k}_{0}^{*}}{\tilde{v}_{0}^{*}} \tilde{w}_{0} .
$$

On the other hand, starting from

$$
b_{+}=\lambda_{b} b_{0}+\lambda_{a} a_{0}+\epsilon \tilde{v}_{L}^{*} \mathfrak{l}_{0} N_{0}
$$

instead of (36), one obtains by analogous calculations that

$$
\lambda_{b}=\frac{\tilde{v}_{L}^{*} \mathfrak{l}_{0}^{*}}{\tilde{w}_{0}^{*}}, \quad \lambda_{a}=-\epsilon \tilde{v}_{L}-\epsilon \frac{\tilde{v}_{L}^{*} \mathfrak{l}_{0}^{*}}{\tilde{w}_{0}^{*}} \tilde{w}_{0} .
$$

Since $\epsilon \tilde{w}_{+}=-\left\langle a_{+}, b_{+}\right\rangle$, it eventually follows that

$$
\begin{aligned}
\tilde{w}_{+}=\frac{\tilde{v}_{L}^{*} \tilde{v}_{R}^{*} \mathfrak{k}_{0}^{*} \mathfrak{l}_{0}^{*}}{\left(\tilde{w}_{0}^{*}\right)^{2}} \tilde{w}_{0}-\frac{\tilde{v}_{L}^{*} \mathfrak{l}_{0}^{*}}{\tilde{w}_{0}^{*}} \tilde{v}_{R}+\frac{\tilde{v}_{R}^{*} \mathfrak{k}_{0}^{*}}{\tilde{w}_{0}^{*}} \tilde{v}_{L}-\epsilon \tilde{v}_{R}^{*} \tilde{v}_{L}^{*} \mathfrak{k}_{0} \mathfrak{l}_{0} \\
+\epsilon^{2} \tilde{w}_{0}\left(-\tilde{v}_{R} \tilde{v}_{L}+\frac{\tilde{v}_{R}^{*} \tilde{v}_{L} \mathfrak{k}_{0}^{*}-\tilde{v}_{R} \tilde{v}_{L}^{*} \mathfrak{l}_{0}^{*}}{\tilde{w}_{0}^{*}} \tilde{w}_{0}+\frac{\tilde{v}_{R}^{*} \tilde{v}_{L}^{*} \mathfrak{k}_{0}^{*} \mathfrak{l}_{0}^{*}}{\left(\tilde{w}_{0}^{*}\right)^{2}} \tilde{w}_{0}^{2}\right)
\end{aligned}
$$

Next, recall that one may consider $(v, w)$ as a function of $(\tilde{v}, \tilde{w})$. More precisely, by (23), one has that $v$ and $w$ approximate $\tilde{v}$ and $\tilde{w}$, respectively, to order $\epsilon^{2}$, in the sense that the family of functions 


$$
\begin{aligned}
(\tilde{v}, \tilde{w}) \mapsto & \left(\epsilon^{-2}(v-\tilde{v}), \epsilon^{-2}(w-\tilde{w})\right) \\
= & \left(\frac{1}{\epsilon^{2}}\left[\epsilon^{-1} \operatorname{arsinh}\left(\epsilon \frac{\tilde{v} \tilde{w}^{*}}{\tilde{v}^{*}}\right)-\tilde{v}\right], \frac{1}{\epsilon^{2}}\left[\epsilon^{-1} \operatorname{arsinh}\left(\epsilon \frac{\tilde{w} \tilde{v}^{*}}{\tilde{w}^{*}}\right)-\tilde{w}\right]\right)
\end{aligned}
$$

is asymptotically analytic on $\mathbb{C}^{2}$, see Definition 6 . Observe further that $\epsilon^{-2}\left(1-\tilde{v}^{*}\right)$ etc. are asymptotically analytic as well. With this, it is straight-forward to conclude (32) from (41).

\subsubsection{Derivation of Equation (33)}

In analogy to (35), one obtains by elementary considerations the following representation of $a_{+}$:

$$
a_{+}=-\epsilon \tilde{w}_{+} b_{+}+\tilde{w}_{+}^{*}\left(b_{+} \times N_{+}\right) .
$$

Using the definition (21) of $\mathfrak{k}$, it then follows that

$$
\left\langle a_{+}, N_{L}\right\rangle=\tilde{w}_{+}^{*}\left\langle b_{+}, N_{+} \times N_{L}\right\rangle=\epsilon \tilde{w}_{+}^{*} \mathfrak{k}_{+}
$$

On the other hand, the computation (36)-(39) implies that

$$
\begin{aligned}
\left\langle a_{+}, N_{L}\right\rangle & =\mu_{b}\left\langle b_{0}, N_{L}\right\rangle+\epsilon \tilde{v}_{R}^{*} \mathfrak{k}_{0}\left\langle N_{0}, N_{L}\right\rangle \\
& =\frac{\mu_{b}}{\tilde{v}_{L}^{*}}\left\langle b_{0}, a_{0} \times b_{+}\right\rangle+\epsilon \tilde{v}_{R}^{*} \mathfrak{l}_{0}^{*} \mathfrak{k}_{0} \\
& =-\frac{\mu_{b} \tilde{w}_{0}^{*}}{\tilde{v}_{L}^{*}}\left\langle b_{+}, N_{0}\right\rangle+\epsilon \tilde{v}_{R}^{*} \mathfrak{l}_{0}^{*} \mathfrak{k}_{0} \\
& =-\epsilon^{2}\left(\tilde{w}_{0}^{*} \tilde{v}_{R}-\tilde{v}_{R}^{*} \mathfrak{k}_{0}^{*} \tilde{w}_{0}\right) \mathfrak{l}_{0}+\epsilon \tilde{v}_{R}^{*} \mathfrak{l}_{0}^{*} \mathfrak{k}_{0} .
\end{aligned}
$$

In combination, this yields

$$
\tilde{w}_{+}^{*} \mathfrak{k}_{+}=\tilde{v}_{R}^{*} \mathfrak{l}_{0}^{*} \mathfrak{k}_{0}+\epsilon\left(\tilde{v}_{R}^{*} \mathfrak{k}_{0}^{*} \tilde{w}_{0}-\tilde{w}_{0}^{*} \tilde{v}_{R}\right) \mathfrak{l}_{0}
$$

We can now substitute (41) to express the unknown $\tilde{w}_{+}$in terms of the known quantities only. Using once again that $\epsilon^{-2}\left(1-\tilde{w}_{+}^{*}\right)$ etc. are asymptotically analytic according to Definition 6, we arrive at (33).

The derivation of Eq. (34) is analogous.

\section{The Abstract Convergence Result}

In this section, we analyze the convergence of solutions to the classical GaussCodazzi system (9)-(12) by solutions to the discrete system (31)-(34). This is the core part of the convergence proof, from which our main result will be easily deduced in the next section. 


\subsection{Domains}

A key concept in the proof is to work with analytic extensions of the quantities $v, w, \mathfrak{k}$ and $\mathfrak{l}$ defined in Sect. 3.4. The analytic setting forces us to introduce yet another class of domains, and corresponding spaces of real analytic functions. In the following, we assume that $r>0$ and $\bar{\rho}>0$ are fixed parameters (which will be frequently omitted in notations), while $h \in(0, \bar{\rho})$ and $\epsilon>0$ may vary, with the restriction that $\epsilon<h$.

For each domain $\Omega(r \mid h)$, introduce its analytic fattening $\widehat{\Omega}_{\bar{\rho}}(r \mid h)$ as follows:

$$
\widehat{\Omega}_{\bar{\rho}}(r \mid h)=\left\{(\xi, \eta) \in \mathbb{C} \times \mathbb{R} ; \exists\left(\xi^{\prime}, \eta\right) \in \Omega(r \mid h) \text { s.t. }\left|\xi-\xi^{\prime}\right| / \bar{\rho}+|\eta| / h<1\right\} .
$$

On these domains, we introduce the function class

$$
C^{\omega}(\widehat{\Omega}(r \mid h)):=\left\{f: \widehat{\Omega}_{\bar{\rho}}(r \mid h) \rightarrow \mathbb{C} ; f(\cdot, \eta) \text { is real analytic, for each } \eta\right\} .
$$

Notice that we require analyticity with respect to $\xi$, but not even continuity with respect to $\eta$. Next, introduce semi-norms $|\cdot|_{\eta, \rho}$ for functions $f \in C^{\omega}(\widehat{\Omega}(r \mid h))$, depending on parameters $\eta \in[-h, h]$ and $\rho \in[0, \bar{\rho}]$ with $\rho / \bar{\rho}+|\eta| / h<1$ as follows:

$$
|f|_{\eta, \rho}=\sup \left\{|f(\xi, \eta)| ; \xi \in \mathbb{C} \text { s.t. } \exists\left(\xi^{\prime}, \eta\right) \in \Omega(r \mid h) \text { with }\left|\xi-\xi^{\prime}\right|<\rho\right\}
$$

These semi-norms are perfectly suited to apply Cauchy estimates; indeed, one easily proves with the Cauchy integral formula that

$$
\left|\partial_{\xi} f\right|_{\eta, \rho} \leq \frac{1}{\rho^{\prime}-\rho}|f|_{\eta, \rho^{\prime}},
$$

provided that $\rho^{\prime}>\rho$. The semi-norms are now combined into a genuine norm $\|\cdot\|_{h}$ on $C^{\omega}(\widehat{\Omega}(r \mid h))$ as follows:

$$
\|f\|_{h}=\sup \left\{\Lambda(\eta, \rho)|f|_{\eta, \rho} ; \frac{|\eta|}{h}+\frac{\rho}{\bar{\rho}}<1\right\},
$$

where the positive weight $\Lambda$ is given by

$$
\Lambda(\eta, \rho)=1-\frac{|\eta| / h}{1-\rho / \bar{\rho}} .
$$

This norm makes $C^{\omega}(\widehat{\Omega}(r \mid h))$ a Banach space.

There is another semi-norm $\{\cdot\}_{h, \delta}$ that will be of importance below: for each $\delta \in[0,1]$, let

$$
\{f\}_{h, \delta}=\sup \left\{|f|_{\eta, \rho} ; \frac{|\eta|}{h}+\frac{\rho}{\bar{\rho}} \leq 1-\delta\right\} .
$$


By definition (44) of the weight $\Lambda$, the following estimate is immediate:

$$
\{f\}_{h, \delta} \leq \delta^{-1}\|f\|_{h},
$$

provided that $\delta>0$.

Replacing $\Omega(r \mid h)$ by $\Omega^{[x y] \epsilon}(r \mid h)$ above yields definitions for analytically fattened domains $\widehat{\Omega}_{\bar{\rho}}^{[x y] \epsilon}(r \mid h)$ with respective spaces $C^{\omega}\left(\widehat{\Omega}^{[x y] \epsilon}(r \mid h)\right)$, semi-norms $|\cdot|_{\eta, \rho}^{[x y] \epsilon}$ and $\{\cdot\}_{h, \delta}^{[x y] \epsilon}$, and norms $\|\cdot\|_{h}^{[x y] \epsilon}$ etc.

\subsection{Statement of the Approximation Result}

Recall that $r>0$ and $\bar{\rho}>0$ are fixed parameters.

Definition 7 An analytic solution $\theta=(v, w, \mathfrak{k}, \mathfrak{l})$ of the classical Gauss-Codazzi system on $\widehat{\Omega}_{\bar{\rho}}(r \mid h)$ consists of four functions $v, w, \mathfrak{k}, \mathfrak{l} \in C^{\omega}(\widehat{\Omega}(r \mid h))$ that are globally bounded on $\widehat{\Omega}_{\bar{\rho}}(r \mid h)$, are continuously differentiable with respect to $\eta$, and satisfy Eqs. (9)-(12) on $\widehat{\Omega}_{\bar{\rho}}(r \mid h)$.

An analytic solution $\theta^{\epsilon}=\left(v^{\epsilon}, w^{\epsilon}, \mathfrak{k}^{\epsilon}, \mathfrak{l}^{\epsilon}\right)$ of the $\epsilon$-discrete Gauss-Codazzi system on $\widehat{\Omega}_{\bar{\rho}}(r \mid h)$ consists of four functions $v^{\epsilon}, w^{\epsilon} \in C^{\omega}\left(\widehat{\Omega}^{[x y] \epsilon}(r \mid h)\right), \mathfrak{k}^{\epsilon} \in$ $C^{\omega}\left(\widehat{\Omega}^{[x x y] \epsilon}(r \mid h)\right), \mathfrak{l}^{\epsilon} \in C^{\omega}\left(\widehat{\Omega}^{[x y y] \epsilon}(r \mid h)\right)$ that satisfy Eqs. (31)-(34) on $\widehat{\Omega}_{\bar{\rho}}^{[x x y y] \epsilon}(r \mid h)$.

A suitable norm to measure the deviation of an $\epsilon$-discrete solution $\theta^{\epsilon}$ to a classical solution $\theta$ on the same domain $\widehat{\Omega}_{\bar{\rho}}(r \mid h)$ is given by the norms of the differences of the four components,

$$
\|\| \theta^{\epsilon}-\theta\|\|_{h}=\max \left(\left\|v^{\epsilon}-v\right\|_{h}^{[x y] \epsilon},\left\|w^{\epsilon}-w\right\|_{h}^{[x y] \epsilon},\left\|\mathfrak{k}^{\epsilon}-\mathfrak{k}\right\|_{h}^{[x x y] \epsilon},\left\|\mathfrak{l}^{\epsilon}-\mathfrak{l}\right\|_{h}^{[x y y] \epsilon}\right) .
$$

Proposition 3 Let an analytic solution $\theta$ to the Gauss-Codazzi system on $\widehat{\Omega}_{\bar{\rho}}(r \mid \bar{h})$ be given, and consider a family $\left(\theta^{\epsilon}\right)_{\epsilon>0}$ of (a priori not necessarily analytic) solutions $\theta^{\epsilon}=\left(v^{\epsilon}, w^{\epsilon}, \mathfrak{k}^{\epsilon}, \mathfrak{l}^{\epsilon}\right)$ to the $\epsilon$-discrete Gauss-Codazzi equations on $\Omega\left(r \mid h^{\epsilon}\right)$. Then there are numbers $A, B>0$ and $\bar{\epsilon}>0$ such that the following is true for all $\epsilon \in(0, \bar{\epsilon})$ : if $\theta^{\epsilon}$ possesses sufficient regularity to admit $\xi$-analytic complex extensions for $\eta$ near zero such that

$$
\left\|\left|\theta-\theta^{\epsilon}\right|\right\|_{\epsilon}<A \epsilon
$$

then $\theta^{\epsilon}$ as a whole extends to an analytic solution $\theta^{\epsilon}$ of the $\epsilon$-discrete Gauss-Codazzi system on $\widehat{\Omega}_{\bar{\rho}}(r \mid h)^{\epsilon}$, and

$$
\left\|\left|\theta-\theta^{\epsilon}\right|\right\|_{h^{\epsilon}} \leq B \epsilon .
$$


Remark 6 The formulation of the proposition suggests that the height $h$ of the domain on which convergence takes place is small. However, this is misleading in general. As it turns out in the proof, the limitation for $h$ is mostly determined by the value of $\bar{\rho}$. In many examples of interest, $\bar{\rho}$ is large compared to the region of interest (determined by $\bar{h}$ and $r$ ), and consequently, one has $h^{\epsilon}=\bar{h}$ above, i.e., convergence takes place on the entire domain of definition of $\theta$.

The rest of this section is devoted to the proof of Proposition 3.

\subsection{Consistency}

We start with an evaluation of the difference between the classical and the $\epsilon$-discrete Gauss-Codazzi equations. Here, we need yet another measure for the deviation of $\theta^{\epsilon}$ from $\theta$ :

$$
\left\{\left\{\theta^{\epsilon}-\theta\right\}\right\}_{h, \delta}=\max \left(\left\{v^{\epsilon}-v\right\}_{h, \delta}^{[x y] \epsilon},\left\{w^{\epsilon}-w\right\}_{h, \delta}^{[x y] \epsilon},\left\{\mathfrak{k}^{\epsilon}-\mathfrak{k}\right\}_{h, \delta}^{[x x y] \epsilon},\left\{\mathfrak{l} \mathfrak{l}^{\epsilon} \mathfrak{l}\right\}_{h, \delta}^{[x y y] \epsilon}\right) .
$$

This semi-norm is similar to $\left\|\mid \theta^{\epsilon}-\theta\right\| \|_{h}$. For further reference, we note that

$$
\left\{\left\{\theta^{\epsilon}-\theta\right\}\right\}_{h, \delta} \leq \frac{1}{\delta}\left|\left\|\theta^{\epsilon}-\theta \mid\right\|_{h}\right.
$$

thanks to (45), provided that $\delta>0$. Furthermore, we denote for abbreviation the difference between corresponding discrete and continuous quantities by $\Delta$, i.e. $\Delta v^{\epsilon}=$ $v^{\epsilon}-v$ etc.

Lemma 5 Let an analytic solution $\theta$ to the classical Gauss-Codazzi system and an analytic solution $\theta^{\epsilon}$ to the $\epsilon$-discrete Gauss-Codazzi system be given, both on $\widehat{\Omega}_{\bar{\rho}}(r \mid h)$. Define the residuals $\tilde{g}_{1}^{\epsilon}, \ldots, \tilde{g}_{4}^{\epsilon} \in C^{\omega}\left(\widehat{\Omega}^{[x x y y] \epsilon}(r \mid h)\right)$ by

$$
\begin{aligned}
\delta_{\eta} \Delta v^{\epsilon} & =\delta_{\xi} \Delta w^{\epsilon}+\epsilon \tilde{g}_{1}^{\epsilon} \\
\delta_{\eta} \Delta w^{\epsilon} & =-\delta_{\xi} \Delta v^{\epsilon}+T_{y}^{-1} \mathfrak{k}^{\epsilon} T_{x}^{-1} \Delta \mathfrak{l}^{\epsilon}+T_{y}^{-1} \Delta \mathfrak{k}^{\epsilon} T_{x}^{-1} \mathfrak{l}+\epsilon \tilde{g}_{2}^{\epsilon} \\
\delta_{y} \Delta \mathfrak{k}^{\epsilon} & =\left(T_{x}^{-1} \mathfrak{l}^{\epsilon}\right)\left(T_{\eta}^{-1} \Delta w^{\epsilon}-T_{\xi} \Delta v^{\epsilon}\right)+\left(T_{x}^{-1} \Delta \mathfrak{l}^{\epsilon}\right)\left(T_{\eta}^{-1} w-T_{\xi} v\right)+\epsilon \tilde{g}_{3}^{\epsilon} \\
\delta_{x} \Delta \mathfrak{l}^{\epsilon} & =\left(T_{y}^{-1} \mathfrak{k}^{\epsilon}\right)\left(T_{\eta}^{-1} \Delta w^{\epsilon}+T_{\xi}^{-1} \Delta v^{\epsilon}\right)+\left(T_{y}^{-1} \Delta \mathfrak{k}^{\epsilon}\right)\left(T_{\eta}^{-1} w+T_{\xi}^{-1} v\right)+\epsilon \tilde{g}_{4}^{\epsilon} .
\end{aligned}
$$

Then the $\tilde{g}_{j}^{\epsilon}$ are uniformly bounded with respect to $\epsilon<\bar{\epsilon}$ on their respective domains:

$$
\left|\tilde{g}_{j}^{\epsilon}\right| \leq G \quad \text { on } \widehat{\Omega}_{\bar{\rho}}^{[x x y y] \epsilon}(r \mid h), \text { for each } j=1, \ldots, 4
$$

with a suitable constant $G$ that depends on $\theta$, and $\theta^{\epsilon}$ only via $\left\{\left\{\theta^{\epsilon}-\theta\right\}\right\}_{h, 0}$, but is independent of $\epsilon$. 
Proof By analyticity of $\theta$ it is clear that the central difference quotients obey

$$
\delta_{\xi} v=\partial_{\xi} v+\epsilon g_{v, \xi}^{\epsilon}, \quad \delta_{\eta} v=\partial_{\eta} v+\epsilon g_{v, \eta}^{\epsilon} \text { etc. }
$$

with functions $g_{v, \xi}^{\epsilon}, g_{v, \eta}^{\epsilon}, \ldots \in C^{\omega}\left(\widehat{\Omega}^{[x x y y] \epsilon}(r \mid h)\right)$ that are bounded uniformly w.r.t. $\epsilon$. The classical Gauss-Codazzi system (9)-(12) thus implies that

$$
\begin{aligned}
\delta_{\eta} v & =\delta_{\xi} w+\epsilon g_{1}^{\epsilon}, \\
\delta_{\eta} w & =-\delta_{\xi} v-\left(T_{y}^{-1} \mathfrak{k}\right)\left(T_{x}^{-1} \mathfrak{l}\right)+\epsilon g_{2}^{\epsilon}, \\
\delta_{y} \mathfrak{k} & =\left(T_{x}^{-1} \mathfrak{l}\right)\left(T_{\eta}^{-1} w-T_{\xi} v\right)+\epsilon g_{3}^{\epsilon}, \\
\delta_{x} \mathfrak{l} & =\left(T_{y}^{-1} \mathfrak{k}\right)\left(T_{\eta}^{-1} w+T_{\xi}^{-1} v\right)+\epsilon g_{4}^{\epsilon},
\end{aligned}
$$

where each of the functions $g_{j}^{\epsilon}$ is bounded on $\Omega^{[x x y y] \epsilon}(r \mid h)$, with an $\epsilon$-independent bound. Taking the difference between each equation of this system and the respective equation of the $\epsilon$-discrete Gauss-Codazzi equation (31)-(34) yields (49)-(52), with

$$
\tilde{g}_{j}^{\epsilon}=h_{j}^{\epsilon}\left(T \theta^{\epsilon}\right)-g_{j}^{\epsilon}
$$

Since the $h_{j}^{\epsilon}$ are asymptotically analytic on $\mathbb{C}^{8}$, it follows that the modulus of $h_{j}^{\epsilon}\left(T \theta^{\epsilon}\right)$ is uniformly controlled on $\Omega^{[x x y y] \epsilon}(r \mid h)$ by the supremum of the modulus of $\left(\theta^{\epsilon}\right)$ 's components.

\subsection{Stability}

Stability is shown inductively. More precisely, we prove for each $n=1,2, \ldots$ with $n \frac{\epsilon}{2} \leq h$ that

$$
\left\|\mid \theta^{\epsilon}-\theta\right\|_{n \frac{\epsilon}{2}}<B \epsilon
$$

In fact, there is nothing to show for $n=1$. For $n=2$, the claim (54) is a consequence of estimate (46) on the initial data. Now assume that (54) has been shown for some $n \geq 2$. We are going to extend the estimate to $n+1$.

Estimate on $\Delta v^{\epsilon}$. We begin by proving the estimate for the $v$-component of $\Delta \theta^{\epsilon}$. Since $v^{\epsilon}$ is defined on $\widehat{\Omega}_{\bar{\rho}}^{[x y] \epsilon}(r \mid h)$, the step $n \rightarrow n+1$ requires to estimate the values of $\Delta v^{\epsilon}\left(\cdot, \eta^{*}\right)$ for $\eta^{*} \in\left((n-1) \frac{\epsilon}{2}, n \frac{\epsilon}{2}\right]$. Choose such an $\eta^{*}$, and define accordingly $\ell$ such that $\eta_{0}^{*}:=\eta^{*}-\ell \epsilon \in\left(-\frac{\epsilon}{2}, \frac{\epsilon}{2}\right]$; in fact, $2 \ell=n$ if $n$ is even, and $n=2 \ell+1$ if $n$ is odd. For $0 \leq k \leq 2 \ell$, introduce

$$
\eta_{k}^{*}=\eta^{*}-(2 \ell-k) \frac{\epsilon}{2}
$$


non-integer values of $k$ are admitted. (55) is consistent with the definition of $\eta_{0}^{*}$, and moreover, $\eta^{*}=\eta_{2 \ell}^{*}$. Using the evolution equation (49), we obtain

$$
\begin{aligned}
\Delta v^{\epsilon}\left(\cdot, \eta^{*}\right) & =\Delta v^{\epsilon}\left(\cdot, \eta_{0}^{*}\right)+\sum_{k=1}^{\ell}\left(T_{\eta} \Delta v^{\epsilon}-T_{\eta}^{-1} \Delta v^{\epsilon}\right)\left(\cdot, \eta_{2 k-1}^{*}\right) \\
& =\Delta v^{\epsilon}\left(\cdot, \eta_{0}^{*}\right)+\epsilon \sum_{k=1}^{\ell} \delta_{\xi} \Delta w^{\epsilon}\left(\cdot, \eta_{2 k-1}^{*}\right)+\epsilon^{2} \sum_{k=1}^{\ell} \tilde{g}_{1}^{\epsilon}\left(\cdot, \eta_{2 k-1}^{*}\right) .
\end{aligned}
$$

Next, pick a $\rho^{*}>0$ such that

$$
\frac{\rho^{*}}{\bar{\rho}}+\frac{\eta^{*}}{h}<1
$$

We estimate:

$$
\begin{aligned}
\left|\Delta v^{\epsilon}\right|_{\eta^{*}, \rho^{*}}^{[x y] \epsilon} \leq & \left|\Delta v^{\epsilon}\right|_{\eta_{0}^{*}, \rho^{*}}^{[x y] \epsilon}+\epsilon \sum_{k=1}^{\ell}\left|\delta_{\xi} \Delta w^{\epsilon}\right|_{\eta_{2 k-1}^{*}, \rho^{*}}^{[x y \xi] \epsilon}+\epsilon^{2} \sum_{k=1}^{\ell}\left|\tilde{g}_{1}^{\epsilon}\right|_{\eta_{2 k-1}^{*}, \rho^{*}}^{[x y y] \epsilon} \\
= & :(\mathrm{I})+(\mathrm{II})+(\mathrm{III}) .
\end{aligned}
$$

We consider the terms (I)-(III) separately. First, thanks to our hypothesis (46) on the initial conditions, we find that

$$
\text { (I) }=\left|\Delta v^{\epsilon}\right|_{\eta_{0}^{*}, \rho^{*}}^{[x y] \epsilon} \leq\left\|\Delta v^{\epsilon}\right\|_{\epsilon}^{[x y] \epsilon} \leq A \epsilon .
$$

Second, recalling the definition of $\|\cdot\|_{h}^{[x y] \epsilon}$, and using a Cauchy estimate (42), we obtain for given $\rho_{2 k-1}^{*}>\rho^{*}$-yet to be determined-

$$
\begin{aligned}
\text { (II) } & =\epsilon \sum_{k=1}^{\ell}\left|\delta_{\xi} \Delta w^{\epsilon}\right|_{\eta_{2 k-1}^{*}, \rho^{*}}^{[x y \xi] \epsilon} \leq \epsilon \sum_{k=1}^{\ell}\left|\partial_{\xi} \Delta w^{\epsilon}\right|_{\eta_{2 k-1}^{*}, \rho^{*}}^{[x y] \epsilon} \leq \epsilon \sum_{k=1}^{\ell} \frac{\left|\Delta w^{\epsilon}\right|_{\eta_{2 k-1}^{*}, \rho_{2 k-1}^{*}}^{[x y] \epsilon}}{\rho_{2 k-1}^{*}-\rho^{*}} \\
& \leq \epsilon\left(\sum_{k=1}^{\ell} \frac{1}{\left(\rho_{2 k-1}^{*}-\rho^{*}\right) \Lambda\left(\eta_{2 k-1}^{*}, \rho_{2 k-1}^{*}\right)}\right)\left\|\Delta w^{\epsilon}\right\|_{n \frac{\epsilon}{2}}^{[x y] \epsilon} .
\end{aligned}
$$

We make the particular choice

$$
\rho_{2 k-1}^{*}:=\frac{\bar{\rho}}{2}\left(1-\frac{\eta_{2 k-1}^{*}}{h}+\frac{\rho^{*}}{\bar{\rho}}\right),
$$


which yields that

$$
\begin{aligned}
\rho_{2 k-1}^{*}-\rho^{*} & =\frac{\bar{\rho}}{2}\left(1-\frac{\eta_{2 k-1}^{*}}{h}-\frac{\rho^{*}}{\bar{\rho}}\right), \\
\Lambda\left(\eta_{2 k-1}^{*}, \rho_{2 k-1}^{*}\right) & =\frac{1-\frac{\eta_{2 k-1}^{*}}{h}-\frac{\rho_{2 k-1}^{*}}{\bar{\rho}}}{1-\frac{\rho_{2 k-1}^{*}}{\bar{\rho}}}=\frac{1-\frac{\eta_{2 k-1}^{*}}{h}-\frac{\rho^{*}}{\bar{\rho}}}{1+\frac{\eta_{2 k-1}^{*}}{h}-\frac{\rho^{*}}{\bar{\rho}}} .
\end{aligned}
$$

And so we obtain

$$
\text { (II) } \leq \frac{2}{\bar{\rho}}\left(1+\frac{\eta^{*}}{h}-\frac{\rho^{*}}{\bar{\rho}}\right)\left(\epsilon \sum_{k=1}^{\ell}\left(1-\frac{\eta_{2 k-1}^{*}}{h}-\frac{\rho^{*}}{\bar{\rho}}\right)^{-2}\right)\left\|\Delta w^{\epsilon}\right\|_{n \frac{\epsilon}{2}}^{[x y] \epsilon} .
$$

To estimate the sum above, define $\varphi:\left(\eta_{0}^{*}, \eta^{*}\right) \rightarrow \mathbb{R}$ by

$$
\varphi(\eta)=\left(1-\frac{\eta}{h}-\frac{\rho^{*}}{\bar{\rho}}\right)^{-2}
$$

Since $\varphi$ is a convex function, Jensen's inequality implies that

$$
\int_{\eta_{2 k-2}^{*}}^{\eta_{2 k}^{*}} \varphi(\eta) \mathrm{d} \eta \geq\left(\eta_{2 k}^{*}-\eta_{2 k-2}^{*}\right) \varphi\left(\frac{1}{\eta_{2 k}^{*}-\eta_{2 k-2}^{*}} \int_{\eta_{2 k-2}^{*}}^{\eta_{2 k}^{*}} \eta \mathrm{d} \eta\right)=\epsilon \varphi\left(\eta_{2 k-1}^{*}\right) .
$$

Hence the sum is bounded from above by the respective integral,

$$
\begin{aligned}
\epsilon \sum_{k=1}^{\ell}\left(1-\frac{\eta_{2 k-1}^{*}}{h}-\frac{\rho^{*}}{\bar{\rho}}\right)^{-2} & \leq \int_{\eta_{0}^{*}}^{\eta_{2 \ell}^{*}}\left(1-\frac{\eta}{h}-\frac{\rho^{*}}{\bar{\rho}}\right)^{-2} \mathrm{~d} \eta \\
& \leq h\left(1-\frac{\eta^{*}}{h}-\frac{\rho^{*}}{\bar{\rho}}\right)^{-1}=\frac{h}{\Lambda\left(\eta^{*}, \rho^{*}\right)\left(1-\rho^{*} / \bar{\rho}\right)}
\end{aligned}
$$

The last term (III) is estimated with the help of the bound (53). However, there is a subtlety: a priori, the constant $G$ there is controlled in terms of $\left\{\left\{\theta^{\epsilon}-\theta\right\}\right\}_{n \frac{\epsilon}{2}, 0}$, but the induction estimate (54) is not sufficient to provide such a uniform bound, due to the weight $\Lambda$. Fortunately, a close inspection of the terms in (III) reveals in combination with (57) that we only need bounds on $\left|\tilde{g}_{j}^{\epsilon}\right|_{\eta, \rho}$ where $\rho / \bar{\rho}<1-\eta^{*} / h-\frac{\epsilon}{2} / h$. It is easily deduced from Lemma 5 that an $\epsilon$-uniform estimate on $\left\{\left\{\theta^{\epsilon}-\theta\right\}\right\}_{n \frac{\epsilon}{2}, \delta}$ with $\delta:=\bar{\rho} / h \frac{\epsilon}{2}>0$ suffices in this case, and the latter is obtained by combining (54) with (48). Enlarging $G$ if necessary, we arrive at

$$
\text { (III) } \leq \epsilon^{2} \sum_{k=1}^{\ell} G=(\epsilon \ell) \epsilon G \leq G h \epsilon
$$


After multiplication of (58) by $\Lambda\left(\eta^{*}, \rho^{*}\right) \leq 1$, we arrive at

$$
\begin{aligned}
\Lambda\left(\eta^{*}, \rho^{*}\right)\left|\Delta v^{\epsilon}\right|_{\eta^{*}, \rho^{*}}^{[x y] \epsilon} & \leq A \epsilon+\frac{2 h}{\bar{\rho}} \frac{1+\eta^{*} / h-\rho^{*} / \bar{\rho}}{1-\rho^{*} / \bar{\rho}}\left\|\Delta w^{\epsilon}\right\|_{n \frac{\epsilon}{2}}^{[x y] \epsilon}+G h \epsilon \\
& \leq\left(A+\frac{4 h}{\bar{\rho}} B+G h\right) \epsilon
\end{aligned}
$$

where we have used the induction hypothesis (54) for estimation of $\left\|\Delta w^{\epsilon}\right\|_{n \frac{\epsilon}{2}}^{[x y] \epsilon}$, and the relation (57) for estimation of the quotient. We have just proven inequality (59) for every $\eta^{*} \in\left((n-1) \frac{\epsilon}{2}, n \frac{\epsilon}{2}\right]$, and for every $\rho^{*} \geq 0$ that satisfies (57). Taking the supremum with respect to these quantities yields

$$
\left\|\Delta v^{\epsilon}\right\|_{(n+1) \frac{\epsilon}{2}}^{[x y] \epsilon} \leq\left(A+\frac{4 h}{\bar{\rho}} B+G h\right) \epsilon .
$$

Estimate on $\Delta w^{\epsilon}$. For estimation of the $w$-component, let $\eta^{*} \in\left((n-1) \frac{\epsilon}{2}, n \frac{\epsilon}{2}\right]$ be given as before, and define $\eta_{k}^{*}$ as in (55). In analogy to (56), we have

$$
\begin{aligned}
\Delta w^{\epsilon}\left(\cdot, \eta^{*}\right) & =\Delta w^{\epsilon}\left(\cdot, \eta_{0}^{*}\right)+\epsilon \sum_{k=1}^{\ell} \delta_{\xi} \Delta v^{\epsilon}\left(\cdot, \eta_{2 k-1}^{*}\right)+\epsilon^{2} \sum_{k=1}^{\ell} \tilde{g}_{2}^{\epsilon}\left(\cdot, \eta_{2 k-1}^{*}\right) \\
& +\epsilon \sum_{k=1}^{\ell}\left(T_{y}^{-1} \mathfrak{k}^{\epsilon} T_{x}^{-1} \Delta \mathfrak{l}^{\epsilon}\right)\left(\cdot, \eta_{2 k-1}^{*}\right)+\epsilon \sum_{k=1}^{\ell}\left(T_{y}^{-1} \Delta \mathfrak{k}^{\epsilon} T_{x}^{-1} \mathfrak{l}\right)\left(\cdot, \eta_{2 k-1}^{*}\right)
\end{aligned}
$$

Taking the $|\cdot|_{\eta^{*}, \rho^{*}}^{[x y] \epsilon}$-norm on both sides, multiplying by $\Lambda\left(\eta^{*}, \rho^{*}\right)<1$, and estimating the first couple of terms as above, we find that

$$
\begin{aligned}
& \Lambda\left(\eta^{*}, \rho^{*}\right)\left|\Delta w^{\epsilon}\right|_{\eta^{*}, \rho^{*}}^{[x y] \epsilon} \leq\left(A+\frac{4 h}{\bar{\rho}} B+G h\right) \epsilon \\
& +\epsilon \sum_{k=1}^{\ell}\left(\left|\mathfrak{k}^{\epsilon}\right|_{\eta_{2 k-\frac{3}{2}}^{*}}^{[x x y] \epsilon}, \rho^{*} \Lambda\left(\eta^{*}, \rho^{*}\right)\left|\Delta \mathfrak{l}^{\epsilon}\right|_{\eta_{2 k-\frac{3}{2}}^{*}, \rho^{*}}^{[x y y] \epsilon}+|\mathfrak{l}|_{\eta_{2 k-\frac{3}{2}}^{*}, \rho^{*}}^{[x y y] \epsilon} \Lambda\left(\eta^{*}, \rho^{*}\right)\left|\Delta \mathfrak{k}^{\epsilon}\right|_{\eta_{2 k-\frac{3}{2}}^{*}\left[\begin{array}{l}
{[x y] \epsilon} \\
\eta^{*}
\end{array}\right.}^{[}\right) \\
& \leq\left(A+\frac{4 h}{\bar{\rho}} B+G h\right) \epsilon+\epsilon \sum_{k=1}^{\ell}\left(\left|\mathfrak{k}^{\epsilon}\right|_{\eta_{2 k-\frac{3}{2}}^{*}}^{[x x y] \epsilon}, \rho^{*}\left\|\Delta \boldsymbol{l}^{\epsilon}\right\|_{n \frac{\epsilon}{2}}^{[x x y] \epsilon}+|\mathfrak{l}|_{\eta_{2 k-\frac{3}{2}}^{*}}^{[x y y] \epsilon}, \rho^{*}\left\|\Delta \mathfrak{k}^{\epsilon}\right\|_{n \frac{\epsilon}{2}}^{[x x y] \epsilon}\right) \text {. }
\end{aligned}
$$

On the one hand, the analytic solution $\theta$ is bounded on $\widehat{\Omega}_{\bar{\rho}}(r \mid \bar{h})$, and so

$$
\|\mathfrak{k}\|_{n \frac{\epsilon}{2}}^{[x x y] \epsilon} \leq \Theta:=\sup _{\widehat{\Omega}_{\bar{\rho}}(r \mid \bar{h})}|\theta| .
$$


On the other hand, since $\eta_{2 k-\frac{3}{2}}^{*} \leq \eta^{*}-\frac{3}{4} \epsilon$, and because of (57), we have that

$$
\Lambda\left(\eta_{2 k-\frac{3}{2}}^{*}, \rho^{*}\right)=\frac{1-\eta_{2 k-\frac{3}{2}}^{*} / h-\rho^{*} / \bar{\rho}}{1-\rho^{*} / \bar{\rho}} \geq \frac{3}{4} \frac{\epsilon}{h},
$$

and therefore, using the induction hypothesis (54),

$$
\begin{aligned}
\left|\mathfrak{k}^{\epsilon}\right|_{\eta_{2 k-\frac{3}{2}}^{*}, \rho^{*}}^{[x x y] \epsilon} \leq|\mathfrak{k}|_{\eta_{2 k-\frac{3}{2}}^{*}, \rho^{*}}^{[x x y] \epsilon}+\left|\Delta \mathfrak{k}^{\epsilon}\right|_{\eta_{2 k-\frac{3}{2}}^{*}\left[\rho^{*}\right.}^{[x x y] \epsilon} & \leq \sup _{\widehat{\Omega}_{\bar{\rho}}(r \mid \bar{h})}|\theta|+\frac{\left\|\Delta \mathfrak{k}^{\epsilon}\right\|_{n \epsilon}^{[x x y] \epsilon}}{\Lambda\left(\eta_{2 k-\frac{3}{2}}^{*}, \rho^{*}\right)} \\
& \leq \Theta+\frac{B \epsilon}{(3 \epsilon) /(4 h)}=\Theta+\frac{4}{3} B h .
\end{aligned}
$$

The remaining terms $\left\|\Delta^{\mathfrak{k} \epsilon}\right\|_{n \frac{\epsilon}{2}}^{[x y y \epsilon}$ and $\left\|\Delta^{\epsilon}\right\|_{n \frac{\epsilon}{2}}^{[x y y] \epsilon}$ in (61) can be estimated directly by (54). Substitution of these partial estimates into (61), and recalling that $\ell \epsilon \leq h$, leads to

$$
\Lambda\left(\eta^{*}, \rho^{*}\right)\left|\Delta v^{\epsilon}\right|_{\eta^{*}, \rho^{*}}^{[x y] \epsilon} \leq\left(A+\left[\frac{4}{\bar{\rho}}+2 \Theta+\frac{4}{3} B h\right] B h+G h\right) \epsilon .
$$

Estimate on $\Delta \mathfrak{k}^{\epsilon}$. Finally, let us estimate $\Delta \mathfrak{k}^{\epsilon}\left(\cdot, \eta^{*}\right)$ at some $\eta^{*} \in\left(\left(n-\frac{3}{2}\right) \frac{\epsilon}{2},(n-\right.$ $\left.\frac{1}{2}\right) \frac{\epsilon}{2}$ ]. For the estimates below, let in addition a $\xi^{*} \in \mathbb{C}$ be given such that $\left(\xi^{*}, \eta^{*}\right) \in$ $\widehat{\Omega}_{\bar{\rho}}^{[x x y] \epsilon}(r \mid h)$. We need to use a slightly different normalization for the $\eta_{k}^{*}$ in (55): write $\eta^{*}=\eta_{-\frac{1}{2}}^{*}+m \frac{\epsilon}{2}$ for suitable $\eta_{-\frac{1}{2}}^{*} \in\left(-\frac{\epsilon}{4}, \frac{\epsilon}{4}\right]$ and a (uniquely determined) $m \in \mathbb{N}$. Now define

$$
\xi_{k}^{*}=\xi^{*}+\left(m-k+\frac{1}{2}\right) \frac{\epsilon}{2}, \quad \eta_{k}^{*}=\eta^{*}-\left(m-k+\frac{1}{2}\right) \frac{\epsilon}{2}
$$

note that $\xi^{*}=\xi_{m-1 / 2}^{*}$ and $\eta^{*}=\eta_{m-\frac{1}{2}}^{*}$. With these notations:

$$
\begin{aligned}
& \Delta \mathfrak{k}^{\epsilon}\left(\xi^{*}, \eta^{*}\right)=\Delta \mathfrak{k}^{\epsilon}\left(\xi_{-\frac{1}{2}}^{*}, \eta_{-\frac{1}{2}}^{*}\right)+\sum_{k=0}^{m-1}\left(T_{y} \Delta \mathfrak{k}^{\epsilon}-T_{y}^{-1} \Delta \mathfrak{k}^{\epsilon}\right)\left(\xi_{k}^{*}, \eta_{k}^{*}\right) \\
& =\Delta \mathfrak{k}^{\epsilon}\left(\xi_{-\frac{1}{2}}^{*}, \eta_{-\frac{1}{2}}^{*}\right)+\epsilon \sum_{k=0}^{m-1} \mathfrak{l}^{\epsilon}\left(\xi_{k+\frac{1}{2}}^{*}, \eta_{k-\frac{1}{2}}^{*}\right)\left(\Delta w^{\epsilon}\left(\xi_{k}^{*}, \eta_{k-1}^{*}\right)-\Delta v^{\epsilon}\left(\xi_{k+1}^{*}, \eta_{k}\right)\right) \\
& +\epsilon \sum_{k=0}^{m-1} \Delta \mathfrak{l}^{\epsilon}\left(\xi_{k+\frac{1}{2}}^{*}, \eta_{k-\frac{1}{2}}^{*}\right)\left(w\left(\xi_{k}^{*}, \eta_{k-1}^{*}\right)-v\left(\xi_{k+1}^{*}, \eta_{k}^{*}\right)\right)+\epsilon^{2} \sum_{k=0}^{m-1} \tilde{g}_{3}^{\epsilon}\left(\xi_{k}^{*}, \eta_{k}^{*}\right) .
\end{aligned}
$$

It is straight-forward to verify that all the terms on the right-hand side are well-defined for the given arguments. For a given $\rho^{*}$ that satisfies (57), we apply the semi-norm $|\cdot|_{\eta^{*}, \rho^{*}}^{[x x y] \epsilon}$ to both sides and estimate further, using the triangle inequality: 


$$
\begin{aligned}
& \left|\Delta \mathfrak{k}^{\epsilon}\right|_{\eta^{*}, \rho^{*}}^{[x x y] \epsilon} \leq\left|\Delta \mathfrak{k}^{\epsilon}\right|_{\eta_{-\frac{1}{2}}^{*}, \rho^{*}}^{[x x y] \epsilon}+\left.\epsilon \sum_{k=0}^{m-1}||^{\epsilon}\right|_{\eta_{k-\frac{1}{2}}^{*}, \rho^{*}} ^{[x x y] \epsilon}\left(\left|\Delta w^{\epsilon}\right|_{\eta_{k-1}, \rho^{*}}^{[x y] \epsilon}+\left|\Delta v^{\epsilon}\right|_{\eta_{k}, \rho^{*}}^{[x y] \epsilon}\right) \\
& +\epsilon \sum_{k=0}^{m-1}\left|\Delta \mathfrak{l}^{\epsilon}\right|_{\eta_{k-1}^{*}, \rho^{*}}^{[x x y] \epsilon}\left(\left.|w|\right|_{\eta_{k-1}, \rho^{*}} ^{[x y]}+|v|_{\eta_{k}^{*}, \rho^{*}}^{[x y] \epsilon}\right)+\epsilon^{2} \sum_{k=0}^{m-1}\left|\tilde{g}_{3}^{\epsilon}\right|_{\eta_{k}^{*}, \rho^{*}}^{[x y]}
\end{aligned}
$$

On the one hand, we have that

$$
|w|_{\eta_{k-1}, \rho^{*}}^{[x y] \epsilon}+|v|_{\eta_{k}, \rho^{*}}^{[x y] \epsilon} \leq \sup _{\widehat{\Omega_{\bar{\rho}}(r \mid \bar{h})}}|w|+\sup _{\widehat{\Omega_{\bar{\rho}}(r \mid \bar{h})}}|v| \leq 2 \Theta,
$$

with the bound $\Theta$ from (62). And on the other hand, arguing like in (63) on grounds of $\eta_{k-\frac{1}{2}}^{*} \leq \eta^{*}-\frac{3}{4} \epsilon$ for all $k=0, \ldots, m-1$, we have the estimate

$$
\left|\mathfrak{l}^{\epsilon}\right|_{\eta_{k-\frac{1}{2}}^{*}, \rho^{*}}^{[x x y] \epsilon} \leq|\mathfrak{l}|_{\eta_{k-\frac{1}{2}}^{*}, \rho^{*}}^{[x x y] \epsilon}+\left|\Delta \mathfrak{l}^{\epsilon}\right|_{\eta_{k-\frac{1}{2}}^{*}, \rho^{*}}^{[x x y] \epsilon} \leq \Theta+\frac{4}{3} B h .
$$

Substitute this into (65) and multiply by $\Lambda\left(\eta^{*}, \rho^{*}\right)$ to obtain

$$
\left\|\Delta \mathfrak{k}^{\epsilon}\right\|_{(n+1) \frac{\epsilon}{2}}^{[x x y] \epsilon} \leq\left(A+4\left[\Theta+\frac{1}{3} B h\right] B h+G h\right) \epsilon .
$$

Estimate on $\Delta \mathfrak{l}^{\epsilon}$. This is completely analogous to the estimate for $\Delta^{\mathfrak{k}}$ above.

Summarizing the results in (60), (64) and (66), we obtain (54) with $n+1$ in place of $n$, for an arbitrary choice of $B>A$, and any corresponding $h>0$ that is sufficiently small to make the coefficients in front of $\epsilon$ in (60), (64) and (66) smaller than $B$. Notice that the implied smallness condition on $h$ is independent of $\epsilon$.

\section{The Continuous Limit of Discrete Isothermic Surfaces}

We are finally in the position to formulate and prove our main approximation result.

\subsection{From Björling Data to Cauchy Data and Back}

Given analytic Björling data $(f, n)$ in the sense of Theorem 1, first compute the associated frame $\Psi^{0}$, the conformal factor $u^{0}$, and the derived quantities $v^{0}, w^{0}, \mathfrak{k}^{0}, \mathfrak{l}^{0}$ as functions on $(-r, r)$ as detailed in the proof there. We claim that, for any sufficiently small $\epsilon>0$, associated Björling data $f^{\epsilon}: \Omega\left(r \mid \frac{\epsilon}{2}\right) \rightarrow \mathbb{R}^{3}$ for construction of an $\epsilon$ discrete isothermic surface can be prescribed such that the following are true: 
(1) The initial surface piece and its tangent vectors are approximated to first order in $\epsilon$,

$$
\begin{aligned}
f^{\epsilon}(\xi, \eta) & =f(\xi)+\mathscr{O}(\epsilon), \\
\delta_{x} f^{\epsilon}(\xi, \eta) & =\exp \left(u^{0}(\xi)\right) \Psi_{1}^{0}(\xi)+\mathscr{O}(\epsilon), \\
\delta_{y} f^{\epsilon}(\xi, \eta) & =\exp \left(u^{0}(\xi)\right) \Psi_{2}^{0}(\xi)+\mathscr{O}(\epsilon),
\end{aligned}
$$

where the $\mathscr{O}(\epsilon)$ indicate $\epsilon$-smallness that is uniform in $(\xi, \eta)$ on the domains $\Omega\left(r \mid \frac{\epsilon}{2}\right)$ for $f^{\epsilon}$, and $\Omega^{[x] \epsilon}\left(r \mid \frac{\epsilon}{2}\right)$ for $\delta_{x} f^{\epsilon}$, and $\Omega^{[y] \epsilon}\left(r \mid \frac{\epsilon}{2}\right)$ for $\delta_{y} f^{\epsilon}$, respectively.

(2) The derived quantities $\left(v^{\epsilon}, w^{\epsilon}, \mathfrak{k}^{\epsilon}, \mathfrak{l}^{\epsilon}\right)$ satisfy

$$
v^{\epsilon}(\xi, \eta)=v^{0}(\xi), \quad \tilde{w}^{\epsilon}(\xi, \eta)=w^{0}(\xi), \quad \mathfrak{k}^{\epsilon}(\xi, \eta)=\mathfrak{k}^{0}(\xi), \quad \mathfrak{l}^{\epsilon}(\xi, \eta)=\mathfrak{l}^{0}(\xi),
$$

at each point $(\xi, \eta)$ in $\Omega^{[x y] \epsilon}(r \mid \epsilon)$ for $v^{\epsilon}, \tilde{w}^{\epsilon}$, in $\Omega^{[x x y] \epsilon}(r \mid \epsilon)$ for $\mathfrak{k}^{\epsilon}$, and in $\Omega^{[x y y] \epsilon}(r \mid \epsilon)$ for $\mathfrak{l}^{\epsilon}$, respectively.

Notice that the data $\left(v^{\epsilon}, w^{\epsilon}, \mathfrak{k}^{\epsilon}, \mathfrak{l}^{\epsilon}\right)$ are $\xi$-analytic quantities; ironically, one cannot even expect continuity of the respective data $f^{\epsilon}$ in general.

For later reference, we briefly sketch one possible construction of such data $f^{\epsilon}$. We start by defining $f^{\epsilon}$ on point triples in the strip $-3 \frac{\epsilon}{4}<\xi \leq 3 \frac{\epsilon}{4}$ : let $(\xi, \eta) \in \Omega\left(r \mid \frac{\epsilon}{2}\right)$ be a point with $-\frac{\epsilon}{4}<\xi \leq \frac{\epsilon}{4}$. We distinguish two cases. If $0<\eta \leq \frac{\epsilon}{2}$, then we define $f^{\epsilon}\left(\xi, \eta-\frac{\epsilon}{2}\right)=f(0)$, and there is a unique way to assign data $f^{\epsilon}$ at the two points $\left(\xi-\frac{\epsilon}{2}, \eta\right)$ and $\left(\xi+\frac{\epsilon}{2}, \eta\right)$ such that for the vectors

$$
a=\frac{1}{\epsilon}\left(f^{\epsilon}\left(\xi+\frac{\epsilon}{2}, \eta\right)-f^{\epsilon}\left(\xi, \eta-\frac{\epsilon}{2}\right)\right), \quad b=\frac{1}{\epsilon}\left(f^{\epsilon}\left(\xi-\frac{\epsilon}{2}, \eta\right)-f^{\epsilon}\left(\xi, \eta-\frac{\epsilon}{2}\right)\right),
$$

the following is true:

(1) $a$ is parallel to $\Psi_{1}^{0}(0)$, and $b$ is orthogonal to $n(0)$,

(2) $\epsilon w^{0}(\xi)=\frac{\langle a, b\rangle}{\|a\|\|b\|}$,

(3) $\|a\|=\exp \left(u^{0}(0)+\frac{\epsilon}{2} v^{0}(\xi)\right)$ and $\|b\|=\exp \left(u^{0}(0)-\frac{\epsilon}{2} v^{0}(\xi)\right)$.

If instead $-\frac{\epsilon}{2}<\eta \leq 0$, then we define $f^{\epsilon}\left(\xi, \eta+\frac{\epsilon}{2}\right)=f(0)$, and we assign data $f^{\epsilon}$ at $\left(\xi+\frac{\epsilon}{2}, \eta\right)$ and at $\left(\xi-\frac{\epsilon}{2}, \eta\right)$ with the respective adaptations for the conditions on the vectors.

Up to here, there has been a certain degree of freedom in the choice of the $f^{\epsilon}$. From now on, there is a unique way to extend the already prescribed $f^{\epsilon}$ to all of $\Omega\left(r \mid \frac{\epsilon}{2}\right)$ such that (68) — and, incidentally, also (67) — holds. We briefly indicate how to proceed in the next step; the further steps are then made inductively in the same way. Let $(\xi, \eta)$ be a point with $3 \frac{\epsilon}{4}<\xi \leq 5 \frac{\epsilon}{4}$, and with $-\frac{\epsilon}{2}<\eta \leq 0$. Note that $f^{\epsilon}$ is already defined at the following points: $(\xi-\epsilon, \eta),\left(\xi-\frac{\epsilon}{2}, \eta+\frac{\epsilon}{2}\right)$ and $\left(\xi-3 \frac{\epsilon}{2}, \eta+\frac{\epsilon}{2}\right)$. Let us introduce the vectors

$$
a=\frac{1}{\epsilon}\left(f^{\epsilon}\left(\xi-\frac{\epsilon}{2}, \eta+\frac{\epsilon}{2}\right)-f^{\epsilon}(\xi-\epsilon, \eta)\right), \quad b=\frac{1}{\epsilon}\left(f^{\epsilon}\left(\xi-3 \frac{\epsilon}{2}, \eta+\frac{\epsilon}{2}\right)-f^{\epsilon}(\xi-\epsilon, \eta)\right) .
$$


Then, there is a unique choice for $f^{\epsilon}(\xi, \eta)$ such that the new vector

$$
c=\frac{1}{\epsilon}\left(f^{\epsilon}\left(\xi-\frac{\epsilon}{2}, \eta+\frac{\epsilon}{2}\right)-f^{\epsilon}(\xi, \eta)\right)
$$

satisfies the following conditions:

(1) the sin-value of the angle between the planes spanned by $(a, b)$ and by $(b, c)$, respectively, equals to $\epsilon \mathfrak{k}^{0}\left(\xi-3 \frac{\epsilon}{4}\right)$,

(2) $\frac{\langle a, c\rangle}{\|a\|\|c\|}=\epsilon w^{0}\left(\xi-\frac{\epsilon}{2}\right)$,

(3) $\|c\|=\|b\| \exp \left(\frac{\epsilon}{2} v^{0}\left(\xi-\frac{\epsilon}{2}\right)\right)$.

By continuing this construction in an inductive manner, we enlarge the domain of definition with respect to $\xi$ by $\frac{\epsilon}{2}$ in both directions in each step, until $f^{\epsilon}$ is defined on all of $\Omega\left(r \mid \frac{\epsilon}{2}\right)$. It is obvious from the construction that (68) holds. The verification of (67) is a tedious but straight-forward exercise in elementary geometry that we leave to the interested reader. An important point is that the aforementioned construction only uses data that can be obtained very directly from the Björling data $(f, n)$. Indeed, the calculation of $u^{0}, \Psi^{0}$ and $\left(v^{0}, w^{0}, \mathfrak{k}^{0}, \mathfrak{l}^{0}\right)$ from $(f, n)$ only involves differentiation and inversion of matrices. In particular, all operations are local.

Definition 8 Assume that analytic Björling data $(f, n)$ and discrete data $f^{\epsilon}: \Omega\left(r \mid \frac{\epsilon}{2}\right)$ are given such that (67) and (68) are satisfied. The maximal $\epsilon$-discrete isothermic surface $F^{\epsilon}: \Omega\left(r \mid h_{\epsilon}\right)$ that is obtained from $f^{\epsilon}$ as Björling data-see Proposition 1 -is referred to as grown from $(f, n)$.

\subsection{Main Result}

The central approximation result is the following.

Theorem 2 Let analytic Björling data $(f, n)$ on $(-r, r)$ be given, and let $F$ : $\Omega(r \mid \bar{h}) \rightarrow \mathbb{R}^{3}$ be the corresponding real-analytic isothermic surface. Further, let $F^{\epsilon}: \Omega\left(r \mid h^{\epsilon}\right) \rightarrow \mathbb{R}^{3}$ be the family of $\epsilon$-discrete isothermic surfaces that are grown from $(f, n)$.

Then, there are some $h>0$ and $C>0$, such that for all sufficiently small $\epsilon>0$, we have that $h_{\epsilon} \geq h$, and

$$
\begin{array}{r}
\left\|F^{\epsilon}(\xi, \eta)-F(\xi, \eta)\right\| \leq C \epsilon, \quad\left\|\delta_{x} F^{\epsilon}(\xi, \eta)-F_{x}(\xi, \eta)\right\| \leq C \epsilon, \\
\left\|\delta_{y} F^{\epsilon}(\xi, \eta)-F_{y}(\xi, \eta)\right\| \leq C \epsilon
\end{array}
$$

for all $(\xi, \eta) \in \Omega^{[x y] \epsilon}(r \mid h)$.

Theorem 2 above gives an answer to the question of how to approximate the unique isothermic surface $F$ that is determined by given Björling data by a family of $\epsilon$ isothermic surfaces $F^{\epsilon}$. Our construction of the surface $F^{\epsilon}$ is completely explicit, 

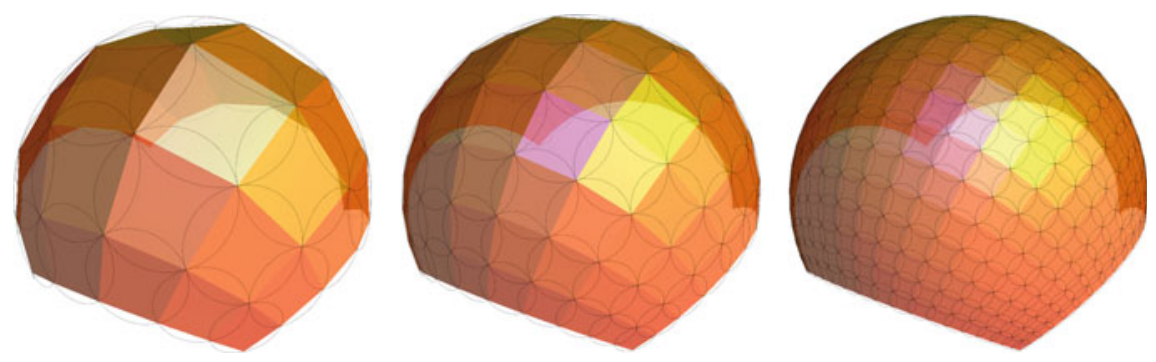

Fig. 5 A part of a sphere in different degrees of discretization
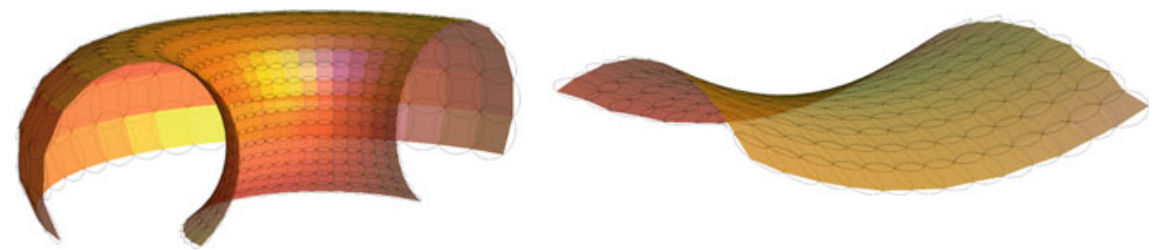

Fig. 6 Examples of discrete isothermic surfaces: torus (left), hyperbolic paraboloid (right)

and it requires no a priori knowledge about $F$. The plots in Figs. 5 and 6 illustrate that our construction can be used to generate pictures of the surfaces $F^{\epsilon}$ with just a few lines of code.

Remark 7 Note that if the discrete isothermic surfaces $F^{\epsilon}$ converge to a smooth isothermic surface $F$, then also the discrete Christoffel and Darboux transforms of $F^{\epsilon}$ converge to the corresponding smooth Christoffel and Darboux transforms of $F$. This will be proven in Sect. 6 .

Proof (of Theorem 2) Since $F$ is real-analytic on $\Omega(r \mid \bar{h})$, the derived quantities $u, v, w$ and $\mathfrak{k}, \mathfrak{l}$ are real-analytic there as well, and can be extended to functions in $C^{\omega}(\widehat{\Omega}(r \mid \bar{h}))$, for a suitable choice of the "fattening parameter" $\bar{\rho}>0$, after diminishing $\bar{h}>0$ if necessary. The extensions satisfy the Gauss-Codazzi system (9)-(12) on the complexified domain.

Next, consider the $\epsilon$-discrete isothermic surfaces $F^{\epsilon}: \Omega\left(r \mid h_{\epsilon}\right) \rightarrow \mathbb{R}$ that are grown from the Björling data $(f, n)$. Define the associated quantities $v^{\epsilon}, w^{\epsilon}, \mathfrak{k}^{\epsilon}, \mathfrak{l}^{\epsilon}$. Thanks to (68), these are real analytic functions on $\Omega^{[x y] \epsilon}(r \mid \epsilon)$, and they extend complex-analytically w.r.t. $\xi$ to $\widehat{\Omega}_{\bar{\rho}}^{[x y] \epsilon}(r \mid \epsilon)$. Since the quadruple $\left(v^{\epsilon}, w^{\epsilon}, \mathfrak{k}^{\epsilon}, \mathfrak{l}^{\epsilon}\right)$ satisfies the discrete Gauss-Codazzi equations (31)-(34), the $\xi$-analyticity is propagated from the initial strip to the maximal domain of existence, i.e., $v^{\epsilon}, w^{\epsilon} \in$ $C^{\omega}\left(\widehat{\Omega}^{[x y] \epsilon}\left(r \mid h_{\epsilon}\right)\right)$ etc.

Moreover, again thanks to (68), the differences $\Delta v^{\epsilon}, \Delta \mathfrak{k}^{\epsilon}$ and $\Delta \mathfrak{l}^{\epsilon}$ are of order $\mathscr{O}(\epsilon)$ on the initial strip:

$$
\left\|\Delta v^{\epsilon}\right\|_{\epsilon}^{[x y] \epsilon} \leq A \epsilon, \quad\left\|\Delta \mathfrak{k}^{\epsilon}\right\|_{\epsilon}^{[x x y] \epsilon} \leq A \epsilon, \quad\left\|\Delta \mathfrak{l}^{\epsilon}\right\|_{\epsilon}^{[x y y] \epsilon} \leq A \epsilon
$$


with a suitable $\epsilon$-independent constant $A$. For the remaining differences $\Delta w^{\epsilon}$, it follows via Lemma 3 on the equivalence of $\left(v^{\epsilon}, w^{\epsilon}\right)$ and $\left(\tilde{v}^{\epsilon}, \tilde{w}^{\epsilon}\right)$ that they satisfy the same estimate (enlarging $A$ if necessary):

$$
\left\|\Delta w^{\epsilon}\right\|_{\epsilon}^{[x y] \epsilon} \leq A \epsilon .
$$

We are thus in the situation to apply Proposition 3. From the estimate (47), it follows in particular that

$$
v^{\epsilon}=v+\mathscr{O}(\epsilon), \quad w^{\epsilon}=w+\mathscr{O}(\epsilon), \quad \mathfrak{k}^{\epsilon}=\mathfrak{k}+\mathscr{O}(\epsilon), \quad \mathfrak{l}^{\epsilon}=\mathfrak{l}+\mathscr{O}(\epsilon) .
$$

Here and below, we use the slightly ambiguous notation $\mathscr{O}(\epsilon)$ to express that the discrete quantities approximate the associated continuous ones uniformly on their respective (real) domains $\Omega^{[x y] \epsilon}\left(r \mid h_{\epsilon}\right)$ or $\Omega^{[x x y] \epsilon}\left(r \mid h_{\epsilon}\right), \Omega^{[x y y] \epsilon}\left(r \mid h_{\epsilon}\right)$, with a maximal error of order $\epsilon$.

Next, we conclude from (70) that also

$$
a^{\epsilon}=a+\mathscr{O}(\epsilon), \quad b^{\epsilon}=b+\mathscr{O}(\epsilon), \quad \hat{u}^{\epsilon}=u+\mathscr{O}(\epsilon), \quad \check{u}^{\epsilon}=u+\mathscr{O}(\epsilon) .
$$

Indeed, it follows directly from the definition of these quantities that (71) implies

$$
\delta_{x} F^{\epsilon}=\exp \left(\hat{u}^{\epsilon}\right) a^{\epsilon}=\exp (u+\mathscr{O}(\epsilon))(a+\mathscr{O}(\epsilon))=\exp (u) a+\mathscr{O}(\epsilon)=\partial_{x} F+\mathscr{O}(\epsilon)
$$

and, likewise, $\delta_{y} F^{\epsilon}=\partial_{y} F+\mathscr{O}(\epsilon)$, which, eventually, implies further that also $F^{\epsilon}=F+\mathscr{O}(\epsilon)$, thanks to $F^{\epsilon}(\xi, \eta)=F(0)$ for $-\frac{\epsilon}{4}<\xi<\frac{\epsilon}{4}$ and $-\frac{\epsilon}{2}<\eta \leq \frac{\epsilon}{2}$ by construction. Therefore, our claim (69) is a consequence of (71).

We only sketch the proof of (71), that is little more than a repeated application of the Gronwall lemma. For further technical details, we refer the reader to [2], where the relevant estimates have been carried out in a very similar situation, see the proof of Theorem 5.4 therein.

First of all, the claim (71) holds on the strip $\Omega^{[x y] \epsilon}(r \mid \epsilon)$ thanks to (67). Now compare the frame equations (6) \& (7) with their discrete analogs from (28)-(30):

$$
\begin{aligned}
& \partial_{x} u=w+v \text { and } \delta_{x} \check{u}^{\epsilon}=w^{\epsilon}+v^{\epsilon}, \\
& \partial_{y} u=w-v \text { and } \delta_{y} \hat{u}^{\epsilon}=w^{\epsilon}-v^{\epsilon}, \\
& \partial_{x} b=(w-v)\left(T_{y}^{-1} a+\mathscr{O}(\epsilon)\right) \text { and } \delta_{x} b^{\epsilon}=\left(w^{\epsilon}-v^{\epsilon}+\mathscr{O}(\epsilon)\right) T_{y}^{-1} a^{\epsilon}+\mathscr{O}(\epsilon) T_{x}^{-1} b^{\epsilon}, \\
& \partial_{y} a=(w-v)\left(T_{x}^{-1} b+\mathscr{O}(\epsilon)\right) \text { and } \delta_{y} a^{\epsilon}=\left(w^{\epsilon}-v^{\epsilon}+\mathscr{O}(\epsilon)\right) T_{x}^{-1} b^{\epsilon}+\mathscr{O}(\epsilon) T_{y}^{-1} a^{\epsilon} .
\end{aligned}
$$

Subtract the respective equations, recall (70), and use a standard Gronwall argument to conclude that the validity of (71) extends from the "initial strip" to the entire domain $\Omega^{[x x y y] \epsilon}(r \mid h)$.

A posteriori, we conclude that $h_{\epsilon} \uparrow h$ as $\epsilon \downarrow 0$. where $h>0$ is the constant obtained in the proof of Proposition 3. Indeed, thanks to the uniform closeness of the discrete tangent vectors $\delta_{x} F^{\epsilon}, \delta_{y} F^{\epsilon}$ to their continuous counterparts $\partial_{x} F, \partial_{y} F-$ 
which are orthogonal with non-vanishing length-it easily follows that there cannot occur any degeneracies in $F^{\epsilon}$ at any $h^{\epsilon}<h$.

\section{Transformations}

Isothermic surfaces have an exceptionally rich transformation theory. For the definition of discrete isothermic surfaces used in this paper this transformation theory carries over to the discrete setup.

We consider two important transformations, namely the Christoffel transformation and Darboux transformation. Their analogs for discrete isothermic surfaces may for example be found in [3, 4, 14-16]. It is a natural question whether the convergence results of Theorem 2 can be generalized to imply the convergence of the transformed surfaces.

\subsection{Christoffel Transformation}

We briefly remind the classical definition of the Christoffel transformation. The included existence claim was first proved by Christoffel [10].

Definition 9 Let $F: \Omega(r \mid h) \rightarrow \mathbb{R}^{3}$ be an isothermic surface. Then the $\mathbb{R}^{3}$-valued one-form $d F^{\star}$ defined by

$$
F_{x}^{\star}=\frac{F_{x}}{\left\|F_{x}\right\|^{2}}, \quad F_{y}^{\star}=-\frac{F_{y}}{\left\|F_{y}\right\|^{2}},
$$

is closed. The surface $F^{\star}: \Omega(r \mid h) \rightarrow \mathbb{R}^{3}$, defined (up to translation) by integration of this one-form, is isothermic and is called dual to the surface $F$ or Christoffel transform of the surface $F$.

Given any isothermic surface $F$ and its dual $F^{\star}$, straightforward calculation leads to the following relations between corresponding quantities.

$$
\begin{aligned}
& F_{x}^{\star}=\mathrm{e}^{-2 u} F_{x}, \quad F_{y}^{\star}=-\mathrm{e}^{-2 u} F_{y}, \quad N^{\star}=-N, \\
& u^{\star}=-u, \quad v^{\star}=-v, \quad w^{\star}=-w, \quad \mathfrak{k}^{\star}=-\mathfrak{k}, \quad \mathfrak{l}^{\star}=\mathfrak{l} .
\end{aligned}
$$

The discrete case is nearly the same, see for example [3]. 
Definition 10 Let $F^{\epsilon}: \Omega(r \mid h) \rightarrow \mathbb{R}^{3}$ be a discrete isothermic surface. Then the $\mathbb{R}^{3}$-valued discrete one-form $\delta\left(F^{\star}\right)^{\epsilon}$ defined by

$$
\delta_{x}\left(F^{\star}\right)^{\epsilon}=\frac{\delta_{x} F^{\epsilon}}{\left\|\delta_{x} F^{\epsilon}\right\|^{2}}, \quad \delta_{y}\left(F^{\star}\right)^{\epsilon}=-\frac{\delta_{y} F^{\epsilon}}{\left\|\delta_{y} F^{\epsilon}\right\|^{2}},
$$

is closed. The surface $\left(F^{\star}\right)^{\epsilon}: \Omega(r \mid h) \rightarrow \mathbb{R}^{3}$, defined (up to translation) by integration of this discrete one-form, is a discrete isothermic surface and is called dual to $F^{\epsilon}$ or Christoffel transform of $F^{\epsilon}$.

Corollary 1 Under the assumptions of Theorem 2 not only the discrete isothermic surface itself converges to the corresponding smooth isothermic surface, but also the discrete Christoffel transforms converge to the corresponding Christoffel transforms of the smooth isothermic surface.

Proof Recall the definitions of the discrete quantities in Sect. 3.4. We immediately deduce the following relations for a discrete isothermic surface $F^{\epsilon}$ and its dual $\left(F^{\star}\right)^{\epsilon}$. For better reading we omit the superscript $\epsilon$.

$$
\begin{aligned}
& a^{\star}=a, \quad b^{\star}=-b, \quad \hat{u}^{\star}=-\hat{u}, \quad \check{u}^{\star}=-\check{u}, \quad N^{\star}=-N, \\
& \tilde{v}^{\star}=-\tilde{v}, \quad \tilde{w}^{\star}=-\tilde{w}, \quad v^{\star}=-v, \quad w^{\star}=-w, \quad \mathfrak{k}^{\star}=-\mathfrak{k}, \quad \mathfrak{l}^{\star}=\mathfrak{l} .
\end{aligned}
$$

Now the proof follows directly from the corresponding proofs in Sects. 4 and 5 .

\subsection{Darboux Transformation}

The Darboux transformation for isothermic surfaces was introduced by Darboux [12]. It is a special case of a Ribaucour transformation and is closely connected to Möbius geometry as well as to the integrable system approach to isothermic surfaces, see for example [15].

Definition 11 Let $F: \Omega(r \mid h) \rightarrow \mathbb{R}^{3}$ be an isothermic surface. Then the isothermic surface $F^{+}: \Omega(r \mid h) \rightarrow \mathbb{R}^{3}$ is called a Darboux transform of $F$ if

$$
\begin{aligned}
& F_{x}^{+}=-\frac{\left\|F^{+}-F\right\|^{2}}{C\left\|F_{x}\right\|^{2}}\left(F_{x}-2\left\langle F_{x}, \frac{F^{+}-F}{\left\|F^{+}-F\right\|}\right\rangle \frac{F^{+}-F}{\left\|F^{+}-F\right\|}\right), \\
& F_{y}^{+}=\frac{\left\|F^{+}-F\right\|^{2}}{C\left\|F_{y}\right\|^{2}}\left(F_{y}-2\left\langle F_{y}, \frac{F^{+}-F}{\left\|F^{+}-F\right\|}\right\rangle \frac{F^{+}-F}{\left\|F^{+}-F\right\|}\right),
\end{aligned}
$$

where $C \in \mathbb{R}, C \neq 0$, is a constant which is called parameter of the Darboux transformation.

In the discrete case the definition of the Darboux transformation may be interpreted as "discrete Ribaucour transformation" using intersecting instead of touching spheres. 
In particular, recall the definition of the cross-ratio $q\left(p_{1}, p_{2}, p_{3}, p_{4}\right)$ of four coplanar points $p_{1}, \ldots, p_{4}$. After identification of their common plane with the complex plane $\mathbb{C}$ the cross-ratio may be calculated by the formula

$$
q\left(p_{1}, p_{2}, p_{3}, p_{4}\right):=\left(p_{1}-p_{2}\right)\left(p_{2}-p_{3}\right)^{-1}\left(p_{3}-p_{4}\right)\left(p_{4}-p_{1}\right)^{-1}
$$

The following definition first appeared in [16], see also [4, 15].

Definition 12 Let $F^{\epsilon}: \Omega(r \mid h) \rightarrow \mathbb{R}^{3}$ be a discrete isothermic surface. Then the discrete isothermic surface $\left(F^{+}\right)^{\epsilon}: \Omega(r \mid h) \rightarrow \mathbb{R}^{3}$ is called a discrete Darboux transform of $F^{\epsilon}$ if the following conditions are satisfied.

(i) The four points $T_{x}^{-1} F^{\epsilon}, T_{x} F^{\epsilon}, T_{x}^{-1}\left(F^{+}\right)^{\epsilon}, T_{x}\left(F^{+}\right)^{\epsilon}$ lie in a common plane and the same is true for $T_{y}^{-1} F^{\epsilon}, T_{y} F^{\epsilon}, T_{y}^{-1}\left(F^{+}\right)^{\epsilon}, T_{y}\left(F^{+}\right)^{\epsilon}$.

(ii) $q\left(T_{x}^{-1} F^{\epsilon}, T_{x} F^{\epsilon}, T_{x}\left(F^{+}\right)^{\epsilon}, T_{x}^{-1}\left(F^{+}\right)^{\epsilon}\right)=\frac{1}{\gamma}$ and $q\left(T_{y}^{-1} F^{\epsilon}, T_{y} F^{\epsilon}, T_{y}\left(F^{+}\right)^{\epsilon}, T_{y}^{-1}\left(F^{+}\right)^{\epsilon}\right)=-\frac{1}{\gamma}$, where $\gamma \in \mathbb{R}, \gamma \neq 0$ is a constant which is called parameter of the Darboux transformation.

Note that given any discrete isothermic surface, a discrete Darboux transform may be obtained by prescribing the value of $\left(F^{+}\right)^{\epsilon}$ at one point and using the conditions of the definition to successively build a new surface which is also discrete isothermic (as long as the surface does not degenerate).

In order to obtain convergence of the discrete Darboux transform to the corresponding continuous one, we choose $\gamma=C / \epsilon^{2}$.

Corollary 2 Under the assumptions of Theorem 2 not only the discrete isothermic surface itself converges to the corresponding smooth isothermic surface, but also the discrete Darboux transforms (with $\gamma=C / \epsilon^{2}$ ) converge to the corresponding Darboux transforms (with parameter $C$ ) of the smooth isothermic surface.

Proof Assume that the discrete isothermic surface itself converges to the corresponding smooth isothermic surface with errors of order $\mathscr{O}(\epsilon)$ as in the proofs of Theorem 2. Now start with $\left(F^{+}\right)^{\epsilon}(0,0)=F^{+}(0,0)$ and build the discrete Darboux transform successively using the above definition. Denote the distance between corresponding points by $d^{\epsilon}=\left(F^{+}\right)^{\epsilon}-F^{\epsilon}$.

In order to relate corresponding discrete and smooth quantities, we first use the simple equivalence

$$
\frac{\left(p_{2}-p_{1}\right)\left(p_{4}-p_{3}\right)}{\left(p_{3}-p_{2}\right)\left(p_{1}-p_{4}\right)}=\frac{1}{q} \quad \Longleftrightarrow \quad p_{3}-p_{2}=\frac{\left(p_{4}-p_{1}\right)-\left(p_{2}-p_{1}\right)}{1-q \frac{\left(p_{4}-p_{1}\right)}{\left(p_{2}-p_{1}\right)}}
$$


Then we use the fact that in our case $\left(p_{2}-p_{1}\right)=\mathscr{O}(\epsilon)$ and $q=C / \epsilon^{2}$. Thus we obtain that

$$
p_{3}-p_{2}=\frac{\left(p_{4}-p_{1}\right)-\epsilon \frac{\left(p_{2}-p_{1}\right)}{\epsilon}}{1-\frac{\epsilon^{2}\left(p_{4}-p_{1}\right)}{C\left(p_{2}-p_{1}\right)}}=\left(p_{4}-p_{1}\right)+\epsilon\left(\frac{\left(p_{4}-p_{1}\right)^{2}}{C \frac{\left(p_{2}-p_{1}\right)}{\epsilon}}-\frac{\left(p_{2}-p_{1}\right)}{\epsilon}\right)+\mathscr{O}\left(\epsilon^{2}\right) .
$$

Now we identify

$$
\left(p_{3}-p_{2}\right)=T_{x} d^{\epsilon}, \quad\left(p_{4}-p_{1}\right)=T_{x}^{-1} d^{\epsilon}, \quad \frac{\left(p_{2}-p_{1}\right)}{\epsilon}=\delta_{x} F^{\epsilon}
$$

and easily deduce by straightforward identifications of complex numbers and vectors that

$$
\begin{aligned}
\frac{T_{x} d^{\epsilon}-T_{x}^{-1} d^{\epsilon}}{\epsilon} & =\frac{1}{C\left\|\delta_{x} F^{\epsilon}\right\|^{2}}\left(-\left\|T_{x}^{-1} d^{\epsilon}\right\|^{2} \delta_{x} F^{\epsilon}+2 T_{x}^{-1} d^{\epsilon}\left\langle T_{x}^{-1} d^{\epsilon}, \delta_{x} F^{\epsilon}\right\rangle\right)-\delta_{x} F^{\epsilon}+\mathscr{O}(\epsilon) \\
& =F_{x}^{+}-F_{x}+\mathscr{O}(\epsilon) .
\end{aligned}
$$

Analogously, we obtain

$$
\begin{aligned}
\frac{T_{y} d^{\epsilon}-T_{y}^{-1} d^{\epsilon}}{\epsilon} & =\frac{1}{C\left\|\delta_{y} F^{\epsilon}\right\|^{2}}\left(\left\|T_{y}^{-1} d^{\epsilon}\right\|^{2} \delta_{y} F^{\epsilon}-2 T_{y}^{-1} d^{\epsilon}\left\langle T_{y}^{-1} d^{\epsilon}, \delta_{y} F^{\epsilon}\right\rangle\right)-\delta_{y} F^{\epsilon}+\mathscr{O}(\epsilon) \\
& =F_{y}^{+}-F_{y}+\mathscr{O}(\epsilon)
\end{aligned}
$$

Thus starting with $\left(F^{+}\right)^{\epsilon}(0,0)=F^{+}(0,0)$ and building the discrete Darboux transform successively using the above definition, in each step we add an error of order $\mathscr{O}\left(\epsilon^{2}\right)$ to the difference $d=F^{+}-F$ of the Darboux pair. Therefore we obtain $\left(F^{+}\right)^{\epsilon}=F^{+}+\mathscr{O}(\epsilon)$ as claimed.

Remark 8 Using the definitions of continuous and discrete Darboux transformations, corresponding formulas for $a^{+}, b^{+}, \hat{u}^{+}, \check{u}^{+}, N^{+}, \tilde{v}^{+}, \tilde{w}^{+}, v^{+}, w^{+}, \mathfrak{k}^{+}, \mathfrak{l}^{+}$may be deduced, which also converge under the assumptions of Theorem 2.

Acknowledgments The authors would like to thank Sepp Dorfmeister and Fran Burstall for stimulating and helpful discussions. We further thank the anonymous referee for the careful reading of the initial manuscript and various suggestions for improvement. This research was supported by the DFG Collaborative Research Center TRR 109 "Discretization in Geometry and Dynamics".

Open Access This chapter is distributed under the terms of the Creative Commons AttributionNoncommercial 2.5 License (http://creativecommons.org/licenses/by-nc/2.5/) which permits any noncommercial use, distribution, and reproduction in any medium, provided the original author(s) and source are credited.

The images or other third party material in this chapter are included in the work's Creative Commons license, unless indicated otherwise in the credit line; if such material is not included in the work's Creative Commons license and the respective action is not permitted by statutory regulation, users will need to obtain permission from the license holder to duplicate, adapt or reproduce the material. 


\section{References}

1. Bobenko, A.I., Matthes, D., Suris, Y.B.: Discrete and smooth orthogonal systems: $C^{\infty}$ approximation. Int. Math. Res. Not. 45, 2415-2459 (2003)

2. Bobenko, A.I., Matthes, D., Suris, Y.B.: Nonlinear hyperbolic equations in surface theory: integrable discretizations and approximation results. St. Petersburg Math. J. 17, 39-61 (2006)

3. Bobenko, A.I., Pinkall, U.: Discrete isothermic surfaces. J. reine angew. Math. 475, 187-208 (1996)

4. Bobenko, A.I., Suris, Y.B.: Discrete differential geometry. Integrable structure. In: Graduate Studies in Mathematics, vol. 98. AMS (2008)

5. Brander, D., Dorfmeister, J.F.: The Björling problem for non-minimal constant mean curvature surfaces. Commun. Anal. Geom. 18(1), 171-194 (2010)

6. Bücking, U.: Approximation of conformal mapping by circle patterns. Geom. Dedicata 137, 163-197 (2008)

7. Burstall, F.: Isothermic surfaces: conformal geometry, Clifford algebras and integrable systems. In: Terng, C.L. (ed.) Integrable Systems, Geometry and Topology, AMS/IP Studies in Advanced Math., vol. 36, pp. 1-82. American Mathematical Society (2006)

8. Burstall, F., Hertrich-Jeromin, U., Pedit, F., Pinkall, U.: Curved flats and isothermic surfaces. Math. Z. 225(2), 199-209 (1997)

9. Cayley, A.: On the determination of the surfaces divisible into squares by means of their curves of curvature. Proc. London Math. Soc. 4, 8-9 (1872)

10. Christoffel, E.: Ueber einige allgemeine Eigenschaften der Minimumsflächen. Crelles J. 67, 218-228 (1867)

11. Cieśliński, J., Goldstein, P., Sym, A.: Isothermic surfaces in $\mathbf{E}^{3}$ as soliton surfaces. Phys. Lett. A 205(1), 37-43 (1995)

12. Darboux, G.: Sur les surfaces isothermiques. Comptes Rendus 128(1299-1305), 1538 (1899)

13. Dierkes, U., Hildebrandt, S., Küster, A., Wohlrab, O.: Minimal surfaces. I, Grundlehren der Mathematischen Wissenschaften [Fundamental Principles of Mathematical Sciences], vol. 295. Springer-Verlag, Berlin (1992)

14. Hertrich-Jeromin, U.: Transformations of discrete isothermic nets and discrete cmc-1 surfaces in hyperbolic space. Manuscr. Math. 102, 465-486 (2000)

15. Hertrich-Jeromin, U.: Introduction to Möbius differential geometry. In: London Mathematical Society Lecture Note Series, vol. 300. Cambridge University Press, Cambridge (2003)

16. Hertrich-Jeromin, U., Hoffmann, T., Pinkall, U.: A discrete version of the Darboux transform for isothermic surfaces. In: Bobenko, A.I., Seiler, R. (eds.) Discrete Integrable Geometry and Physics. Oxford Lecture Ser. Math. Appl, vol. 16, pp. 59-81. Clarendon Press, Oxford (1999)

17. Matthes, D.: Convergence in discrete Cauchy problems and applications to circle patterns. Conform. Geom. Dyn. 9, 1-23 (2005)

18. Nagumo, M.: Über das Anfangswertproblem partieller Differentialgleichungen. Jap. J. Math. 18, 41-47 (1942)

19. Nirenberg, L.: An abstract form of the nonlinear Cauchy-Kowalewski theorem. J. Differ. Geom. 6, 561-576 (1972)

20. Schief, W.K.: Isothermic surfaces in spaces of arbitrary dimension: integrability, discretization, and Bäcklund transformations - a discrete Calapso equation. Stud. Appl. Math. 106(1), 85-137 (2001)

21. Schramm, O.: Circle patterns with the combinatorics of the square grid. Duke Math. J. 86(2), 347-389 (1997)

22. Stephenson, K.: Introduction to circle packing: the theory of discrete analytic functions. Cambridge University Press, New York (2005)

23. Walter, W.: An elementary proof of the Cauchy-Kowalevsky theorem. Amer. Math. Monthly 92(2), 115-126 (1985)

24. Weingarten, J.: Über die Differentialgleichung der Oberflächen, welche durch ihre Krümmungslinien in unendlich kleine Quadrate geteilt werden können. Sitzungsber. königl. preuß. Akad. Wiss. pp. 1163-1166 (1883) 